\title{
Turn of the Month Effect across Equity Markets, Across Industries, and Across Extreme Market Conditions
}

\author{
Samique March-Dallas* \\ School of Business and Industry \\ Florida A\&M University, Tallahassee, FL \\ Shahid Hamid \\ College of Business Administration \\ Florida International University, Miami, FL
}

\begin{abstract}
This study looks at the turn of the month anomaly that was first researched by Ariel (1987). We look at successive sub periods since the late 1980s, and in the context of the 2007US financial crisis to investigate whether the turn of the month effect is present across the US market, similar across industry indices, and different in bull and bear markets as well as in periods of extreme market conditions. The turn of the month effect is present in only some of the breadth of market indices in the US but is very evident in the industry indices. Periods of extreme market conditions, like the downturn in 2007, do not show this turn of the month effect. While the turn of the month effect is present across the entire sample period for some indices, the earlier sub-period up to 1998 seems to account for much of this observation.
\end{abstract}

Keywords:_Turn of the month; market anomaly; equity markets

\section{Introduction}

The traditional paradigm in Finance is heavily dependent on the Efficient Market Hypothesis proposed by Fama (1972) and the asset pricing models proposed by Sharpe (1964), Lintner (1965) and Black (1972). Empirical research has shown that there are deviations from this pre-accepted notion and these deviations are anomalous to this school of thought. Anomalies such as the size effect, momentum, January effect, weekend effect and turn of the month effect have been shown to persist. A variety of explanations have been presented but there appears to be no consensus to either their existence or their causality. This study looks at the turn of the month anomaly that was first researched by Ariel (1987) and further examined by Lakonishok and Smidt (1988). We look at successive sub periods since the late 1980s, and in the context of the 2007 US financial crisis. We investigate whether the effect is present across the US market, similar across industry indices, and different in bull and bear markets as well as in periods of extreme market conditions. Such examination can shed light on the causes and consequences of the turn of the month effect.

This study looks at data post the period - 1897 to 1986 - that Lakonishok and Smidt concentrated on. The data starts from prior to the crash of 1989, goes through the financial turmoil of 2008 and ends in June 2010. Since past studies have been criticized for possible data snooping [Sullivan, Timmermann and White (1998)], a new dataset and time period is selected here. The dataset consists of 11 indices - the Dow Jones Industrial Average (DJIA), the Russell 3000, the Russell 2000, the Russell 1000, the S\&P 500, the NASDAQ Composite, the NASDAQ 100, and five industry indices.

This paper differs from Lakonishok and Smidt (1988) as well as Ariel (1987) in that it is not concentrated on one market index, and the possible industry effect is taken into account. Furthermore, recent periods are evaluated and an attempt is made to differentiate periods of extreme markets conditions. There are six market representative indices used to illustrate the effect of the turn of the month on the entire market. There are also several industry indices that are used to see if the turn of the month is industry specific. All three Russell Indices as well as the NASDAQ 100 are included to ascertain if there is a size effect that might also be influential. The turn of the month effect is pronounced for the industry indices such as the DJU and DJT but is less pronounced for the broader market indices such as the DJIA. Furthermore, the sub-period, 1989-1998, accounts for much of the effect exhibited over the entire period. For the bear market during the financial crisis, on the average, most of the losses accrued in the first few days of the month. This crisis period shows the least amount of significance and no turn of the month effect. The rest of the paper is organized in the following manner. The next section discusses prior literature that has looked at the turn of the month anomaly, followed by a section on data, methodology, results and conclusion. 


\section{Literature Review}

Ariel (1987) was the first researcher to find the existence of the monthly effect. He found that the magnitude of the monthly effect is pronounced and it is comparable to that of the weekend effect. The cumulative advancement of the market can be attributed to the first half of the month while the second half of the month contributed negatively. Lakonishok and Smidt (1988) further extended on Ariel's work by showing that the monthly effect was not confined to the entire first half of the month but actually is evident in the last trading day of the previous month to the first four trading days of the following month, hence the new name - the turn of the month effect. Thaler (1987) agrees that aside from the turn of the month the DJIA does not measure up, in fact it falls.

Reinganum (1984) concludes that prevailing asset pricing theories cannot explain the anomalies. Therefore, the existence of anomalies is a call to theorists to pursue new paths that can better explain the equities market. Agrawal and Tandon (1994) find that the turn-of-the- month effect is not a uniquely US phenomenon and is present in at least fourteen countries worldwide. This observance further backs the idea that the theory is lagging behind the empirical studies. Holden, Thompson, Ruangrit (2005) allow that some explanation for observed variation in the markets can be attributed to calendar anomalies. Hansen, Lunde and Nason (2005) also find evidence of calendar effects but more so on small cap and mid-size indices, indicating there is also a size effect evident within the turn of the month effect. Therefore, any study looking at anomalies should also account for the size effect.

Booth, Kallunki, Martikainen (2001) verify the existence of the turn of the month in Finnish stocks and then purport a link between the turn of the month effect to liquidity. Liquidity is increased particularly in the turn of the month period when compared to the rest of the month. Jaffe and Westerfield (1989) while they do not find strong evidence for the monthly effect proposed by Ariel, they do find strong evidence for an effect centered around the last trading day of the month which is consistent with the turn of the month effect. Boudreaux (1995) extends Jaffe and Westerfield's study by looking at an additional seven countries and they found the existence of the turn of the month effect as well. Kunkel, Compton and Beyer (2003) find that the turn of the month period on average can be attributed with $87 \%$ of the monthly return which agrees with Agrawal and Tandon's findings. However, it is unclear whether the effect is present across all domestic markets, similar across industry indices, and different in bull and bear markets as well as in periods of extreme market conditions.

\section{Data}

The data used is daily data on the following eleven indices: the DJIA, Dow Jones Transportation, Dow Jones Utilities, S\&P500, NASDAQ Composite, NASDAQ 100, NASDAQ Transportation, NASDAQ Industrial, Russell 3000, Russell 2000, and Russell 1000. Dividing the data into the market representative indices plus the industry indices will give more insight into whether this turn of the month observance is relegated only to the market index or if there is an industry specific component as well. Using the three Russell indices will also allow us to account for the size effect. In particular, the Russell 3000 index represents approximately 98\% of the total market value for all stocks traded on US exchanges. The Russell 3000 is reconstituted every year in June and stocks are chosen by sorting all US stocks by market capitalization adjusted by number of outstanding shares then the top 3000 are chosen to be a part of the index. The Russell 2000 index is composed of the small cap stocks while the Russell 1000 is composed of the 1000 large cap stocks.

The S\&P 500 index has been used often as a measure of the market benchmark folder even though it contains less stock than the Russell 3000. Due to its prominence it is included in the study. The DJIA is comprised of thirty stocks, however while this small number does not adequately cover the number or market capitalization of total US equity it does give a unique indication of the current trends in the market. The DJIA is the longest published market index in the USA and is well established with both retail and institutional investors who use it to estimate investor sentiment. The NASDAQCompositeis useful in analysis as it illustrates the impact of the anomaly on the NASDAQ market where a number of prominent dealer stocks are traded through dealers.

The time period studied is from January 3, 1989 to June 30, 2010 for a total of twenty one and a half years. There are five thousand four hundred and nineteen observations over the entire period. Most of the indices have data for this entire period but the NASDAQ Industrial and NASDAQ Transportation report data as of October 25, 1990 while the Russell 1000 data goes back to December 10, 1992. The data was collected from Yahoo! Finance. The data is divided into three sub-groups. The first sub-group goes from 1989 to 1998 and the second from 1999 to 2010as well as a subgroup looking at the crisis period 2007-2010. 


\section{Methodology}

The methodology used is based on Lakonishok and Smidt's refinement of Ariel's methodology but with additional data since they only focused on the equally-weighted index. The close price data is collected from Yahoo! Finance for all twelve indices. If there was a trading day that was missing for a particular index then the close price was calculated by extrapolating from the previous day and the next day close prices. The price data was assigned a trading day for each day in the trading month based on whether the trade took place in the first half or the second half of the month. Trading days in the first half of the month were numbered up to positive twelve and trading days in the last half of the month were numbered up to negative eleven. Most months had an even number of trading days but in the event that there was an odd number of trading days then the first half of the month was assigned the extra trading day. Both the percentage rates of return and the logarithmic rates of return were calculated in the following manner ${ }^{1}$.

$$
\begin{aligned}
& \% \text { return }=\frac{P_{t}-P_{t-1}}{P_{t-1}} ; \\
& \text { Ln return }=\ln \left(\frac{P_{t}}{P_{t-1}}\right) \\
& \text { Where, } P_{t} \text { is the current price } \\
& P_{t-1} \text { is the prior day's price }
\end{aligned}
$$

After sorting based on trading days it was seen that the number of observations was consistent for the first seven and last seven trading days of the month so those limits were chosen for reporting the daily rates of returns. The returns of the last seven trading days of the month and the first seven trading days of the month are looked at to observe the pattern for these fourteen days around the turn of the month. Days -7 to -1 are the last seven trading days of the monthwith-1 representing the last day - and days 1 to 7 are the first seven trading days - with 1 representing the first trading day. To test the hypothesis that these means are significantly different from zero, a t-test is employed and the significance at the 5\% level is observed. The sign test and the sign rank test were also used as non-parametric tests of location in contrast to the t-test. For the percent of positive returns a binomial proportion test is employed to test the probability of a fifty percent return. The return for the four day turn of the month return in particular was noted.

Ariel first showed there was a significant difference between the returns pertaining to the first and second half of the trading month. Thus, the data is divided into the first and second halves of the trading month. A trading month on average consists of twenty trading days, so the first half has ten trading days and the second half has ten as well. There are some months that have more than twenty trading days but only the first ten days and the last ten days were used to form the halves. Also, in some instances the trading month had less than twenty trading days so the first half had ten and the second half had nine trading days. The means for each half were evaluated using the t-test to see if the means significantly differed from zero. The sign test and the signed rank tests of location were also performed on the semimonthly returns. Additionally, the binomial proportions test was calculated on the percentage of positive returns for each half.

Lakonishok and Smidt pointed out that there are only four trading days that contribute to the positive return that Ariel found. Therefore, the first five days and the last five days of the trading month are analyzed separately to see if there is a significant difference between the means. The four tests - t-test, sign test, signed rank test, and binomial proportions test - previously used on the daily returns and the semi-monthly returns were again used. This analysis is extended further to look at the first three trading days of the month and the last three trading days of the month to see if there is any significant difference between the means.

\section{Results}

Results are presented in tables 1 through 18 . The tables pertain to all eleven indices. The tables present the frequency, mean, median and standard deviation of the returns for daily, semi-monthly, weekly and three day returns for the periods 1989-2010, 1989-1998, 1999-2010, and 2007-2010. The latter sub-period encompasses the recent period of financial crisis. Additionally, the results of the four tests conducted - t-test, sign test, signed rank test, and binomial proportions test - are also reported for the daily, weekly, three day and semi-monthly returns.

Tables one, two and three show the daily rates of return for the DJIA, DJT and the DJU respectively. All three tables show a positive significant return on the first trading day of the month for all three indices over the entire sample period. This positive significance is also present in two of the three sub-periods, but the 2007-2010 sub-period does not exhibit this pattern.

\footnotetext{
${ }^{1}$ However, all results reported are based on the log rates of return. Results for the percentage rates of returns are available on request.

132
} 
The second trading day also shows positive significance for the DJIA and DJT but for the DJU, this significance is present on the third trading day. The negative significant result previously noted on the last trading day of the month is not present for these three indices. While the DJIA has a negative mean return on the last trading day, this return does not significantly differ from zero. Both the DJU and the DJT have positive significant mean returns on the last trading day of the month. The second to last day of the month is positive significant for the DJT while the DJU has positive significance on the third to last trading day. The significance is observed for the entire sample period but when looking at the sub-periods, it appears that the first sub-period, 1989-1998, is largely responsible for the results over the entire period. The second sub-period, 1999-2010, has no significance but for the first trading day of the month.

The NASDAQ composite index exhibits a strong turn of the month effect with positive significance for the last two and first two days of the month. The entire sample period has significance for the three of the four tests but in the subperiod, 1989-1998, all four tests are strongly significant over these four days. Interestingly, the NASDAQ100 shows negative significance on the first trading day for all periods but 2007-2010. However, there is a turn of the month effect present when looking at the Binomial proportions test. Alternately, the S\&P500 shows positive significance for the first day through all periods but 2007-2010. Except for the last trading day, there is significance from at least one of the four tests for the last three days as well as the first two trading days. The sub-period, 1989-1998, strongly illustrates this with two of the tests.

The Russell 3000 and Russell 1000 exhibits significance for the last three trading days except for the last day as well as the first two trading days using at least two of the four tests. However, it is the small cap, Russell 2000 that shows the strongest support for the turn of the month effect. The Russell 2000 extends the turn of the month effect to a wider range beginning with the fourth to last trading day and ending on the fourth trading day. The least amount of significance is seen on the third trading day of the month. The pattern from the Russell 2000 shows that the turn of the month includes the fourth to last trading day to the fourth trading day with limited significance on the third trading day. The two NASDAQ industry indices - Transportation and Industrial - also show significance around turn of the month. However, the significance on the first trading day is noticeably absent in the first sub-period, 1989-1998, for both indices.

When comparing the indices using semi-monthly returns, the industry indices except for the DJT indicate a positive significance in the second half of the month unlike the DJIA, which indicates positive significance in the first half of the month. The other broad market indices show positive significance for both halves of the month and doesn't favor one half over the other. The significance virtually disappears in the second sub-period, 1999-2010, and is non-existent in the third sub-period, 2007-2010. Alternatively, using the weekly returns to compare the first and the last week returns for the month reveal a pronounced positive significance for the last week in all five of the industry indices. This significance is present in all periods except 2007-2010. On the other hand, the DJIA exhibits no significant preference for the returns for either the first or the last week. The NASDAQ100, Russell 3000 and the S\&P500 are the only three of the other six broad market indices that exhibit a preference for the returns in the last week. The other three broad market indices provide mixed significant results for both weeks. Once again, the sub-period, 2007-2010 does not exhibit any significance.

Further decomposing the weekly return into three day returns reveals slightly different results for the twelve indices. The DJIA as well as the Russell 1000 show no significance for any of the periods. The NASDAQ shows preference for the entire sample but has mixed significance in the first sub-period, 1989-1998, and no significance for the other two sub-periods. The NASDAQ100 only shows preference for the last three days in the sub-period, 1989-1998, while the S\&P500 and the Russell 3000 still shows preference for the last three days over the entire period and 1989-1998. Furthermore, the Russell 2000 and the NASDAQ Transportation both show mixed significant results for both three day periods. While the DJT still favors the last three days, two of the four tests reveal significance for the first three days. The DJU and NASDAQ Industrial still distinctly favor the last three days return.

\section{Conclusion}

It is clear that while the turn of the month effect is pronounced for the industry indices such as the DJU and DJT it is present but less pronounced for the broader market indices such as the DJIA. Furthermore, the effect changes intertemporally over the sub periods with the sub-period, 1989-1998, accounting for much of the effect exhibited over the entire period. For the period of the severe bear market during the financial crisis, on the average, most of the losses accrue in the first few days of the month. This crisis period shows the least amount of significance and no turn of the month effect. 


\section{References}

R. Ariel. “A Monthly Effect in Stock Returns.” Journal of Financial Economics 18 (1987), 161-174

A. Agrawal and K. Tandon. "Anomalies or Illusions? Evidence from Stock Markets in Eighteen Countries." Journal of International Money and Finance 13 (1994), 083-106

G. Booth, J. Kallunki and T. Martikainen. "Liquidity and the turn-of-the-month effect: evidence from Finland." Journal of International Financial Markets, Institutions and Money 11 (2001), 137-146

D. Boudreaux. "The Monthly Effect in International Stock Markets: Evidence and Implications." Journal of Financial and Strategic Decisions 8 (1995),

P. Hansen, A. Lunde, and J. Nason. "Testing the Significance of Calendar Effects" Federal Reserve bank of Atlanta Working Paper Series (2005)

K. Holden, J. Thompson and Y. Ruangrit. "The Asian Crisis and Calendar Effects on Stock Returns in Thailand." European Journal of Operational Research 163 (2005), 242-252

J. Jaffe and R. Westerfield. "Is There a Monthly Effect in Stock Market Returns?: Evidence from Foreign Countries." Journal of Banking and Finance, 1989, 13 (2), 237-244

R. Kunkel, W. Compton and S. Beyer. "The turn-of-the-month effect still lives: the international evidence." International Review of Financial Analysis 12 (2003), 207-221

J. Lakonishok and S. Smidt. "Are Seasonal Anomalies Real? A Ninety Year Perspective." The Review of Financial Studies 1 (1988), 403-425

M. Reinganum. "Discussion: What the Anomalies Mean" The Journal of Finance, Vol. 39, No. 3, Papers and Proceedings, Forty-Second Annual Meeting, American Finance Association, San Francisco, CA, December 28-30, 1983 (Jul., 1984), pp. 837-840

R. Sullivan, A. Timmermann and H. White. "Dangers of Data-Driven Inference: The Case of Calendar Effects in Stock Returns." 1998, University of California San Diego Department of EconomicsWorking Paper

R. Thaler. "Anomalies: Seasonal Movements in Security Prices II: Weekend, Holiday, Turn of the Month, and Intraday Effects." The Journal of Economic Perspectives 1 (1987), 169-177 


\section{Table 1: Dow Jones Industrial Average (DJIA) Daily Rates of Return}

The daily rates of return are shown for the first seven and the last seven trading days of the month. The first seven trading days are depicted as positive numbers where 1 is the first trading day and the last seven trading days are depicted by negative numbers where -1 is the last day. The rates of return are calculated as the log of the ratio of the previous day and the current day. Student-t as well as the non-parametric tests - sign, signed rank and binomial proportions-is also reported. $*$ - significance at the $95 \%$ level

\begin{tabular}{|c|c|c|c|c|c|c|c|c|c|c|c|c|c|c|c|}
\hline & Trading Day & -7 & -6 & -5 & -4 & -3 & -2 & -1 & 1 & 2 & 3 & 4 & 5 & 6 & 7 \\
\hline 1989 & \# of Obs. & 258 & 258 & 258 & 258 & 258 & 258 & 258 & 258 & 258 & 258 & 258 & 258 & 258 & 258 \\
\hline to & Mean & $-0.135 \%$ & $-0.052 \%$ & $0.025 \%$ & $0.126 \%$ & $0.055 \%$ & $0.065 \%$ & $-0.069 \%$ & $0.280 \%$ & $0.120 \%$ & $-0.023 \%$ & $-0.042 \%$ & $0.004 \%$ & $-0.018 \%$ & $-0.073 \%$ \\
\hline \multirow[t]{6}{*}{2010} & Median & $-0.050 \%$ & $-0.025 \%$ & $0.043 \%$ & $0.033 \%$ & $0.101 \%$ & $0.098 \%$ & $-0.043 \%$ & $0.280 \%$ & $0.137 \%$ & $-0.029 \%$ & $0.062 \%$ & $-0.003 \%$ & $0.016 \%$ & $0.039 \%$ \\
\hline & Std deviation & $1.171 \%$ & $1.158 \%$ & $1.099 \%$ & $1.189 \%$ & $0.994 \%$ & $1.156 \%$ & $0.974 \%$ & $1.214 \%$ & $1.029 \%$ & $0.995 \%$ & $1.152 \%$ & $1.047 \%$ & $1.040 \%$ & $1.168 \%$ \\
\hline & Student-t & -1.846 & -0.727 & 0.369 & 1.706 & 0.884 & 0.902 & -1.135 & $3.704 *$ & 1.878 & -0.375 & -0.580 & 0.063 & -0.277 & -1.010 \\
\hline & Sign & -8.500 & -4.000 & 6.500 & 5.000 & 14.000 & 14.000 & -7.000 & $35.500^{*}$ & 15.500 & -6.500 & 7.000 & -1.000 & 1.500 & 4.000 \\
\hline & $\begin{array}{l}\text { Signed Rank } \\
\% \text { Positive }\end{array}$ & $-2350.5^{*}$ & -884.5 & 1014.5 & 1294.5 & 1970.5 & 2119.5 & -1126.5 & $6081.5^{*}$ & $2701.5^{*}$ & -502.5 & 514.5 & 230.5 & 253.5 & -589.5 \\
\hline & Days & 0.469 & 0.484 & 0.527 & 0.519 & $0.554^{*}$ & $0.554^{*}$ & 0.473 & $0.640 *$ & $0.562 *$ & 0.477 & 0.527 & 0.496 & 0.508 & 0.516 \\
\hline 1989 & \# of Obs. & 120 & 120 & 120 & 120 & 120 & 120 & 120 & 120 & 120 & 120 & 120 & 120 & 120 & 120 \\
\hline to & Mean & $-0.133 \%$ & $0.093 \%$ & $0.025 \%$ & $0.061 \%$ & $0.085 \%$ & $0.103 \%$ & $-0.073 \%$ & $0.327 \%$ & $0.181 \%$ & $-0.062 \%$ & $-0.007 \%$ & $0.062 \%$ & $-0.035 \%$ & $0.081 \%$ \\
\hline \multirow[t]{6}{*}{1998} & Median & $-0.050 \%$ & $0.049 \%$ & $0.123 \%$ & $-0.008 \%$ & $0.101 \%$ & $0.108 \%$ & $0.009 \%$ & $0.247 \%$ & $0.157 \%$ & $-0.080 \%$ & $0.016 \%$ & $-0.025 \%$ & $0.028 \%$ & $0.055 \%$ \\
\hline & Std deviation & $0.849 \%$ & $0.926 \%$ & $1.048 \%$ & $0.849 \%$ & $0.797 \%$ & $0.799 \%$ & $0.966 \%$ & $0.920 \%$ & $0.869 \%$ & $0.715 \%$ & $0.784 \%$ & $0.874 \%$ & $0.785 \%$ & $0.869 \%$ \\
\hline & Student-t & -1.722 & 1.104 & 0.260 & 0.788 & 1.162 & 1.411 & -0.824 & $3.898^{*}$ & $2.285^{*}$ & -0.945 & -0.096 & 0.775 & -0.485 & 1.017 \\
\hline & Sign & -2.500 & 1.000 & 9.000 & 0.000 & 9.000 & 10.000 & 1.000 & $19.500^{*}$ & 10.500 & -5.500 & 1.000 & -4.000 & 2.500 & 7.000 \\
\hline & $\begin{array}{l}\text { Signed Rank } \\
\text { \% Positive }\end{array}$ & -472 & 234 & 578 & 222 & 709 & 677 & -15 & $1442 *$ & $958^{*}$ & -427 & 173 & 162 & 156 & 495 \\
\hline & Days & 0.483 & 0.508 & $0.575 *$ & 0.500 & $0.575 *$ & $0.583^{*}$ & 0.508 & $0.667^{*}$ & $0.592 *$ & 0.458 & 0.508 & 0.467 & 0.525 & 0.558 \\
\hline 1999 & \# of Obs. & 138 & 138 & 138 & 138 & 138 & 138 & 138 & 138 & 138 & 138 & 138 & 138 & 138 & 138 \\
\hline to & Mean & $-0.136 \%$ & $-0.179 \%$ & $0.026 \%$ & $0.183 \%$ & $0.029 \%$ & $0.032 \%$ & $-0.065 \%$ & $0.239 \%$ & $0.067 \%$ & $0.010 \%$ & $-0.072 \%$ & $-0.046 \%$ & $-0.003 \%$ & $-0.207 \%$ \\
\hline \multirow[t]{6}{*}{2010} & Median & $-0.054 \%$ & $-0.058 \%$ & $-0.024 \%$ & $0.065 \%$ & $0.089 \%$ & $0.052 \%$ & $-0.089 \%$ & $0.400 \%$ & $0.050 \%$ & $-0.012 \%$ & $0.098 \%$ & $0.022 \%$ & $-0.005 \%$ & $-0.051 \%$ \\
\hline & Std deviation & $1.395 \%$ & $1.318 \%$ & $1.146 \%$ & $1.421 \%$ & $1.141 \%$ & $1.396 \%$ & $0.983 \%$ & $1.424 \%$ & $1.151 \%$ & $1.188 \%$ & $1.397 \%$ & $1.177 \%$ & $1.222 \%$ & $1.365 \%$ \\
\hline & Student-t & -1.142 & -1.597 & 0.262 & 1.513 & 0.296 & 0.268 & -0.781 & $1.971 *$ & 0.687 & 0.101 & -0.604 & -0.460 & -0.032 & -1.786 \\
\hline & Sign & -6.000 & -5.000 & -2.500 & 5.000 & 5.000 & 4.000 & -8.000 & $16.000^{*}$ & 5.000 & -1.000 & 6.000 & 3.000 & -1.000 & -3.000 \\
\hline & $\begin{array}{l}\text { Signed Rank } \\
\text { \% Positive }\end{array}$ & -677.5 & -703.5 & -62.5 & 442.5 & 283.5 & 421.5 & -528.5 & $1608.5^{*}$ & 371.5 & 164.5 & 36.5 & -34.5 & 24.5 & -871.5 \\
\hline & Days & 0.457 & 0.464 & 0.486 & 0.536 & 0.536 & 0.529 & 0.442 & $0.616^{*}$ & 0.536 & 0.493 & 0.543 & 0.522 & 0.493 & 0.478 \\
\hline 2007 & \# of Obs. & 42 & 42 & 42 & 42 & 42 & 42 & 42 & 42 & 42 & 42 & 42 & 42 & 42 & 42 \\
\hline to & Mean & $-0.139 \%$ & $-0.340 \%$ & $-0.106 \%$ & $0.556 \%$ & $0.044 \%$ & $-0.085 \%$ & $-0.126 \%$ & $0.076 \%$ & $0.091 \%$ & $-0.249 \%$ & $-0.428 \%$ & $-0.162 \%$ & $0.203 \%$ & $-0.254 \%$ \\
\hline \multirow[t]{6}{*}{2010} & Median & $0.097 \%$ & $-0.005 \%$ & $-0.112 \%$ & $0.113 \%$ & $0.089 \%$ & $0.076 \%$ & $-0.097 \%$ & $0.476 \%$ & $-0.046 \%$ & $-0.087 \%$ & $0.190 \%$ & $0.083 \%$ & $0.048 \%$ & $0.041 \%$ \\
\hline & Std deviation & $1.928 \%$ & $1.439 \%$ & $1.446 \%$ & $2.070 \%$ & $1.170 \%$ & $1.871 \%$ & $1.289 \%$ & $1.925 \%$ & $1.366 \%$ & $1.418 \%$ & $1.755 \%$ & $1.598 \%$ & $1.350 \%$ & $2.066 \%$ \\
\hline & Student-t & -0.468 & -1.530 & -0.476 & 1.740 & 0.245 & -0.293 & -0.632 & 0.254 & 0.431 & -1.140 & -1.579 & -0.656 & 0.973 & -0.796 \\
\hline & Sign & 1.0 & -1.0 & -3.0 & 4.0 & 2.0 & 2.0 & -3.0 & 5.0 & 0.0 & -2.0 & 3.0 & 5.0 & 2.0 & 1.0 \\
\hline & $\begin{array}{l}\text { Signed Rank } \\
\text { \% Positive }\end{array}$ & -36.5 & -73.5 & -70.5 & 121.5 & 34.5 & 18.5 & -80.5 & 116.5 & -5.5 & -78.5 & -53.5 & 17.5 & 65.5 & -35.5 \\
\hline & Days & 0.524 & 0.476 & 0.429 & 0.595 & 0.548 & 0.548 & 0.429 & 0.619 & 0.500 & 0.452 & 0.571 & 0.619 & 0.548 & 0.524 \\
\hline
\end{tabular}




\section{Table 2: Dow Jones Transportation (DJT) Daily Rates of Return}

The daily rates of return are shown for the first seven and the last seven trading days of the month. The first seven trading days are depicted as positive numbers where 1 is the first trading day and the last seven trading days are depicted by negative numbers where -1 is the last day. The rates of return are calculated as the log of the ratio of the previous day and the current day. Student-t as well as the non-parametric tests - sign, signed rank and binomial proportions- is also reported. $*$ - significance at the $95 \%$ level

\begin{tabular}{|c|c|c|c|c|c|c|c|c|c|c|c|c|c|c|c|}
\hline & Trading Day & -7 & -6 & -5 & -4 & -3 & -2 & -1 & 1 & 2 & 3 & 4 & 5 & 6 & 7 \\
\hline 1989 & $\#$ of Obs. & 258 & 258 & 258 & 258 & 258 & 258 & 258 & 258 & 258 & 258 & 258 & 258 & 258 & 258 \\
\hline to & Mean & $-0.161 \%$ & $-0.093 \%$ & $-0.067 \%$ & $0.080 \%$ & $0.114 \%$ & $0.205 \%$ & $0.188 \%$ & $0.208 \%$ & $0.245 \%$ & $0.011 \%$ & $-0.016 \%$ & $-0.087 \%$ & $-0.120 \%$ & $-0.124 \%$ \\
\hline \multirow[t]{6}{*}{2010} & Median & $-0.136 \%$ & $-0.062 \%$ & $0.019 \%$ & $-0.022 \%$ & $0.081 \%$ & $0.192 \%$ & $0.162 \%$ & $0.268 \%$ & $0.213 \%$ & $-0.054 \%$ & $0.056 \%$ & $-0.016 \%$ & $-0.047 \%$ & $-0.102 \%$ \\
\hline & Std deviation & $1.609 \%$ & $1.372 \%$ & $1.329 \%$ & $1.506 \%$ & $1.325 \%$ & $1.482 \%$ & $1.302 \%$ & $1.532 \%$ & $1.688 \%$ & $1.443 \%$ & $1.455 \%$ & $1.540 \%$ & $1.633 \%$ & $1.582 \%$ \\
\hline & Student-t & -1.608 & -1.093 & -0.808 & 0.859 & 1.383 & $2.220 *$ & $2.321 *$ & $2.185^{*}$ & $2.334^{*}$ & 0.125 & -0.171 & -0.906 & -1.185 & -1.263 \\
\hline & Sign & $-19.5^{*}$ & -5 & 1 & -4 & 10 & 16 & 16 & $28^{*}$ & $17 *$ & -4 & 6 & -1 & -7.5 & -7 \\
\hline & Signed Rank & -2400.5 & -1000.5 & -1018.5 & 152 & 1749 & $2961.5^{*}$ & $2334 *$ & $3548.5^{*}$ & $3320.5^{*}$ & -153.5 & 906.5 & -1310.5 & -1112 & -1921.5 \\
\hline & \% Positive Days & $0.426 *$ & 0.481 & 0.504 & 0.488 & 0.543 & $0.562^{*}$ & $0.566 *$ & $0.609 *$ & $0.566^{*}$ & 0.484 & 0.523 & 0.496 & 0.477 & 0.473 \\
\hline \multirow{8}{*}{$\begin{array}{l}1989 \\
\text { to } \\
1998\end{array}$} & \# of Obs. & 120 & 120 & 120 & 120 & 120 & 120 & 120 & 120 & 120 & 120 & 120 & 120 & 120 & 120 \\
\hline & Mean & $-0.114 \%$ & $0.033 \%$ & $-0.104 \%$ & $-0.039 \%$ & $0.069 \%$ & $0.219 \%$ & $0.185 \%$ & $0.274 \%$ & $0.281 \%$ & $-0.049 \%$ & $0.022 \%$ & $-0.011 \%$ & $-0.185 \%$ & $0.021 \%$ \\
\hline & Median & $-0.098 \%$ & $-0.064 \%$ & $0.056 \%$ & $-0.108 \%$ & $0.129 \%$ & $0.223 \%$ & $0.162 \%$ & $0.170 \%$ & $0.275 \%$ & $-0.081 \%$ & $0.092 \%$ & $-0.112 \%$ & $-0.085 \%$ & $-0.041 \%$ \\
\hline & Std deviation & $0.979 \%$ & $1.163 \%$ & $1.284 \%$ & $0.988 \%$ & $0.994 \%$ & $1.189 \%$ & $1.082 \%$ & $1.114 \%$ & $1.068 \%$ & $0.960 \%$ & $1.154 \%$ & $1.280 \%$ & $0.994 \%$ & $1.079 \%$ \\
\hline & Student-t & -1.278 & 0.313 & -0.891 & -0.427 & 0.759 & $2.014^{*}$ & 1.868 & $2.692^{*}$ & $2.884^{*}$ & -0.557 & 0.204 & -0.095 & -2.037 & 0.217 \\
\hline & Sign & -7 & -3 & 2 & -7 & 5 & 8 & $12^{*}$ & $13^{*}$ & $13 *$ & -3 & 5 & -5 & -8 & -1 \\
\hline & Signed Rank & -492 & -112 & -115 & -217.5 & 436.5 & 759* & $792.5^{*}$ & $947 *$ & $1116^{*}$ & -33 & 211 & -349 & -598.5 & -151 \\
\hline & \% Positive Days & 0.442 & 0.475 & 0.517 & 0.450 & 0.550 & $0.567^{*}$ & $0.608 *$ & $0.608 *$ & $0.608 *$ & 0.475 & 0.542 & 0.458 & 0.442 & 0.492 \\
\hline \multirow{8}{*}{$\begin{array}{l}1999 \\
\text { to } \\
2010\end{array}$} & \# of Obs. & 138 & 138 & 138 & 138 & 138 & 138 & 138 & 138 & 138 & 138 & 138 & 138 & 138 & 138 \\
\hline & Mean & $-0.202 \%$ & $-0.203 \%$ & $-0.034 \%$ & $0.184 \%$ & $0.153 \%$ & $0.193 \%$ & $0.191 \%$ & $0.151 \%$ & $0.214 \%$ & $0.064 \%$ & $-0.048 \%$ & $-0.153 \%$ & $-0.064 \%$ & $-0.251 \%$ \\
\hline & Median & $-0.154 \%$ & $-0.055 \%$ & $-0.055 \%$ & $0.060 \%$ & $0.067 \%$ & $0.165 \%$ & $0.147 \%$ & $0.344 \%$ & $0.165 \%$ & $-0.029 \%$ & $0.016 \%$ & $0.087 \%$ & $0.007 \%$ & $-0.229 \%$ \\
\hline & Std deviation & $2.005 \%$ & $1.526 \%$ & $1.371 \%$ & $1.839 \%$ & $1.559 \%$ & $1.700 \%$ & $1.470 \%$ & $1.821 \%$ & $2.086 \%$ & $1.760 \%$ & $1.678 \%$ & $1.736 \%$ & $2.034 \%$ & $1.911 \%$ \\
\hline & Student-t & -1.182 & -1.566 & -0.292 & 1.175 & 1.156 & 1.333 & 1.528 & 0.977 & 1.205 & 0.424 & -0.334 & -1.033 & -0.372 & -1.545 \\
\hline & Sign & $-12.5^{*}$ & -2 & -1 & 3 & 5 & 8 & 4 & $15^{*}$ & 4 & -1 & 1 & 4 & 0.5 & -6 \\
\hline & Signed Rank & -717.5 & -445.5 & -321.5 & 322.5 & 445.5 & 725.5 & 410.5 & 784.5 & 589.5 & 38.5 & 223.5 & -337.5 & 29.5 & -798.5 \\
\hline & \% Positive Days & $0.413^{*}$ & 0.486 & 0.493 & 0.522 & 0.536 & 0.558 & 0.529 & $0.609 *$ & 0.529 & 0.493 & 0.507 & 0.529 & 0.507 & 0.457 \\
\hline \multirow{8}{*}{$\begin{array}{l}2007 \\
\text { to } \\
2010\end{array}$} & \# of Obs. & 42 & 42 & 42 & 42 & 42 & 42 & 42 & 42 & 42 & 42 & 42 & 42 & 42 & 42 \\
\hline & Mean & $-0.388 \%$ & $-0.442 \%$ & $-0.295 \%$ & $0.521 \%$ & $0.090 \%$ & $-0.017 \%$ & $0.127 \%$ & $0.060 \%$ & $0.243 \%$ & $-0.273 \%$ & $-0.501 \%$ & $-0.139 \%$ & $0.382 \%$ & $-0.164 \%$ \\
\hline & Median & $-0.083 \%$ & $-0.201 \%$ & $-0.456 \%$ & $0.066 \%$ & $0.463 \%$ & $0.209 \%$ & $-0.139 \%$ & $0.516 \%$ & $0.106 \%$ & $-0.498 \%$ & $0.081 \%$ & $0.200 \%$ & $0.458 \%$ & $0.018 \%$ \\
\hline & Std deviation & $2.826 \%$ & $1.811 \%$ & $1.481 \%$ & $2.525 \%$ & $1.831 \%$ & $2.151 \%$ & $1.711 \%$ & $2.592 \%$ & $2.854 \%$ & $2.093 \%$ & $2.260 \%$ & $2.395 \%$ & $1.785 \%$ & $2.729 \%$ \\
\hline & Student-t & -0.890 & -1.583 & -1.290 & 1.336 & 0.319 & -0.053 & 0.482 & 0.150 & 0.553 & -0.846 & -1.437 & -0.377 & 1.385 & -0.389 \\
\hline & Sign & -5 & -2 & -2 & 2 & 3 & 3 & -1 & 4 & 1 & -4 & 1 & 4 & 5 & 0 \\
\hline & Signed Rank & -101.5 & -99.5 & -122.5 & 61.5 & 24.5 & 30.5 & 1.5 & 66.5 & 31.5 & -87.5 & -55.5 & -1.5 & 121.5 & -15.5 \\
\hline & \% Positive Days & 0.381 & 0.452 & 0.452 & 0.548 & 0.571 & 0.571 & 0.476 & 0.595 & 0.524 & 0.405 & 0.524 & 0.595 & 0.619 & 0.500 \\
\hline
\end{tabular}




\section{Table 3: Dow Jones Utilities (DJU) Daily Rates of Return}

The daily rates of return are shown for the first seven and the last seven trading days of the month. The first seven trading days are depicted as positive numbers where 1 is the first trading day and the last seven trading days are depicted by negative numbers where -1 is the last day. The rates of return are calculated as the log of the ratio of the previous day and the current day. Student-t as well as the non-parametric tests - sign, signed rank and binomial proportions- is also reported. $*$ - significance at the $95 \%$ level

\begin{tabular}{|c|c|c|c|c|c|c|c|c|c|c|c|c|c|c|c|}
\hline & Trading Day & -7 & -6 & -5 & -4 & -3 & -2 & -1 & 1 & 2 & 3 & 4 & 5 & 6 & 7 \\
\hline \multirow{8}{*}{$\begin{array}{l}1989 \\
\text { to } \\
2010\end{array}$} & \# of Obs. & 258 & 258 & 258 & 258 & 258 & 258 & 258 & 258 & 258 & 258 & 258 & 258 & 258 & 258 \\
\hline & Mean & $-0.064 \%$ & $-0.049 \%$ & $0.120 \%$ & $0.134 \%$ & $0.099 \%$ & $0.061 \%$ & $0.126 \%$ & $0.134 \%$ & $0.036 \%$ & $-0.097 \%$ & $-0.188 \%$ & $0.000 \%$ & $-0.052 \%$ & $-0.162 \%$ \\
\hline & Median & $-0.014 \%$ & $0.075 \%$ & $0.085 \%$ & $0.088 \%$ & $0.137 \%$ & $0.073 \%$ & $0.103 \%$ & $0.197 \%$ & $0.063 \%$ & $-0.111 \%$ & $-0.073 \%$ & $0.013 \%$ & $0.044 \%$ & $-0.063 \%$ \\
\hline & Std deviation & $1.216 \%$ & $1.156 \%$ & $1.237 \%$ & $1.151 \%$ & $0.980 \%$ & $1.095 \%$ & $0.929 \%$ & $1.180 \%$ & $1.098 \%$ & $1.129 \%$ & $1.203 \%$ & $1.008 \%$ & $1.049 \%$ & $1.182 \%$ \\
\hline & Student-t & -0.839 & -0.688 & 1.564 & 1.866 & 1.615 & 0.901 & $2.177^{*}$ & 1.817 & 0.527 & -1.377 & $-2.516 *$ & 0.000 & -0.795 & $-2.204^{*}$ \\
\hline & Sign & -4 & 5.5 & 13 & 11.5 & $19 *$ & 12 & 16 & $22 *$ & 8.5 & $-16.5^{*}$ & -10.5 & 3 & 5 & -8.5 \\
\hline & Signed Rank & -528 & -142.5 & 1815 & 1704 & $3123 *$ & 1161 & $2745.5^{*}$ & $3416^{*}$ & 1334.5 & -1546 & -2092.5 & 548 & 318 & -1715 \\
\hline & $\%$ Positive Days & 0.496 & 0.527 & $0.554^{*}$ & 0.550 & $0.578^{*}$ & 0.550 & $0.566^{*}$ & $0.589 *$ & 0.535 & $0.442 *$ & 0.461 & 0.523 & 0.523 & 0.473 \\
\hline \multirow{8}{*}{$\begin{array}{l}1989 \\
\text { to } \\
1998\end{array}$} & \# of Obs. & 120 & 120 & 120 & 120 & 120 & 120 & 120 & 120 & 120 & 120 & 120 & 120 & 120 & 120 \\
\hline & Mean & $-0.011 \%$ & $0.027 \%$ & $0.025 \%$ & $0.084 \%$ & $0.121 \%$ & $0.104 \%$ & $0.162 \%$ & $0.069 \%$ & $0.137 \%$ & $-0.071 \%$ & $-0.094 \%$ & $0.049 \%$ & $-0.075 \%$ & $-0.099 \%$ \\
\hline & Median & $-0.066 \%$ & $0.100 \%$ & $0.089 \%$ & $0.088 \%$ & $0.095 \%$ & $0.111 \%$ & $0.131 \%$ & $0.029 \%$ & $0.060 \%$ & $-0.163 \%$ & $-0.105 \%$ & $0.094 \%$ & $0.000 \%$ & $-0.120 \%$ \\
\hline & Std deviation & $0.684 \%$ & $0.702 \%$ & $0.750 \%$ & $0.601 \%$ & $0.658 \%$ & $0.689 \%$ & $0.783 \%$ & $0.770 \%$ & $0.804 \%$ & $0.706 \%$ & $0.761 \%$ & $0.708 \%$ & $0.713 \%$ & $0.675 \%$ \\
\hline & Student-t & -0.182 & 0.429 & 0.370 & 1.529 & $2.019 *$ & 1.660 & $2.268^{*}$ & 0.986 & 1.862 & -1.099 & -1.346 & 0.752 & -1.158 & -1.602 \\
\hline & Sign & -6 & 5 & 8 & 7.5 & 9 & 9 & 9 & 2 & 5.5 & $-14.5^{*}$ & -6.5 & 6 & 0 & -7.5 \\
\hline & Signed Rank & -226.5 & 188.5 & 230.5 & 438.5 & 787.5* & 620.5 & $865^{*}$ & 231.5 & 556 & -653.5 & -486 & 469.5 & -283.5 & -517.5 \\
\hline & \% Positive Days & 0.475 & 0.550 & $0.575^{*}$ & $0.575^{*}$ & $0.583^{*}$ & $0.583^{*}$ & $0.583^{*}$ & 0.525 & 0.550 & $0.392^{*}$ & 0.450 & $0.575^{*}$ & 0.508 & 0.450 \\
\hline \multirow{8}{*}{$\begin{array}{l}1999 \\
\text { to } \\
2010\end{array}$} & \# of Obs. & 138 & 138 & 138 & 138 & 138 & 138 & 138 & 138 & 138 & 138 & 138 & 138 & 138 & 138 \\
\hline & Mean & $-0.109 \%$ & $-0.116 \%$ & $0.203 \%$ & $0.177 \%$ & $0.079 \%$ & $0.024 \%$ & $0.095 \%$ & $0.189 \%$ & $-0.051 \%$ & $-0.119 \%$ & $-0.271 \%$ & $-0.042 \%$ & $-0.032 \%$ & $-0.217 \%$ \\
\hline & Median & $0.025 \%$ & $0.002 \%$ & $0.080 \%$ & $0.088 \%$ & $0.247 \%$ & $0.032 \%$ & $0.072 \%$ & $0.468 \%$ & $0.110 \%$ & $-0.035 \%$ & $-0.038 \%$ & $-0.053 \%$ & $0.140 \%$ & $-0.007 \%$ \\
\hline & Std deviation & $1.538 \%$ & $1.438 \%$ & $1.539 \%$ & $1.473 \%$ & $1.194 \%$ & $1.355 \%$ & $1.041 \%$ & $1.446 \%$ & $1.297 \%$ & $1.399 \%$ & $1.482 \%$ & $1.211 \%$ & $1.274 \%$ & $1.489 \%$ \\
\hline & Student-t & -0.832 & -0.951 & 1.551 & 1.413 & 0.775 & 0.208 & 1.066 & 1.538 & -0.466 & -1.002 & $-2.147^{*}$ & -0.411 & -0.291 & -1.715 \\
\hline & Sign & 2 & 0.5 & 5 & 4 & 10 & 3 & 7 & $20 *$ & 3 & -2 & -4 & -3 & 5 & -1 \\
\hline & Signed Rank & -108.5 & -293.5 & 671.5 & 437.5 & 706.5 & -34.5 & 540.5 & $1265.5^{*}$ & 46.5 & -285.5 & -615.5 & -171.5 & 373.5 & -360.5 \\
\hline & $\%$ Positive Days & 0.514 & 0.507 & 0.536 & 0.529 & $0.572^{*}$ & 0.522 & 0.551 & $0.645^{*}$ & 0.522 & 0.486 & 0.471 & 0.478 & 0.536 & 0.493 \\
\hline \multirow{8}{*}{$\begin{array}{l}2007 \\
\text { to } \\
2010\end{array}$} & \# of Obs. & 42 & 42 & 42 & 42 & 42 & 42 & 42 & 42 & 42 & 42 & 42 & 42 & 42 & 42 \\
\hline & Mean & $-0.093 \%$ & $-0.519 \%$ & $-0.103 \%$ & $0.510 \%$ & $-0.003 \%$ & $-0.043 \%$ & $-0.147 \%$ & $0.296 \%$ & $-0.016 \%$ & $-0.194 \%$ & $-0.680 \%$ & $0.088 \%$ & $0.143 \%$ & $-0.148 \%$ \\
\hline & Median & $-0.048 \%$ & $0.064 \%$ & $-0.225 \%$ & $0.342 \%$ & $0.204 \%$ & $0.019 \%$ & $-0.151 \%$ & $0.758 \%$ & $0.227 \%$ & $-0.110 \%$ & $-0.292 \%$ & $0.123 \%$ & $0.345 \%$ & $0.148 \%$ \\
\hline & Std deviation & $1.724 \%$ & $1.485 \%$ & $1.870 \%$ & $1.966 \%$ & $1.205 \%$ & $1.542 \%$ & $1.268 \%$ & $1.902 \%$ & $1.414 \%$ & $1.396 \%$ & $1.902 \%$ & $1.421 \%$ & $1.335 \%$ & $1.489 \%$ \\
\hline & Student-t & -0.348 & $-2.265^{*}$ & -0.357 & 1.683 & -0.014 & -0.183 & -0.751 & 1.008 & -0.073 & -0.901 & $-2.316^{*}$ & 0.400 & 0.693 & -0.644 \\
\hline & Sign & -1 & 0 & -4 & 5 & 1 & 1 & -4 & 9* & 0 & -1 & -5 & 2 & 5 & 3 \\
\hline & Signed Rank & -46.5 & -125.5 & -86.5 & $166.5^{*}$ & 31.5 & 5.5 & -60.5 & 139.5 & 22.5 & -19.5 & -155.5 & 59.5 & 107.5 & 30.5 \\
\hline & \% Positive Days & 0.476 & 0.500 & 0.405 & 0.619 & 0.524 & 0.524 & $0.405^{*}$ & 0.714 & 0.500 & 0.476 & 0.381 & 0.548 & 0.619 & 0.571 \\
\hline
\end{tabular}




\section{Table4: NASDAQ Daily Rates of Return}

The daily rates of return are shown for the first seven and the last seven trading days of the month. The first seven trading days are depicted as positive numbers where 1 is the first trading day and the last seven trading days are depicted by negative numbers where -1 is the last day. The rates of return are calculated as the log of the ratio of the previous day

and the current day. Student-t as well as the non-parametric tests - sign, signed rank and binomial proportions- is also reported. * significance at the $95 \%$ level

\begin{tabular}{|c|c|c|c|c|c|c|c|c|c|c|c|c|c|c|c|}
\hline & Trading Day & -7 & -6 & -5 & -4 & -3 & -2 & -1 & 1 & 2 & 3 & 4 & 5 & 6 & 7 \\
\hline \multirow{8}{*}{$\begin{array}{l}1989 \\
\text { to } \\
2010\end{array}$} & \# of Obs. & 258 & 258 & 258 & 258 & 258 & 258 & 258 & 258 & 258 & 258 & 258 & 258 & 258 & 258 \\
\hline & Mean & $-0.191 \%$ & $-0.129 \%$ & $0.057 \%$ & $0.176 \%$ & $-0.042 \%$ & $0.096 \%$ & $0.127 \%$ & $0.167 \%$ & $0.136 \%$ & $0.062 \%$ & $-0.014 \%$ & $-0.006 \%$ & $-0.032 \%$ & $-0.089 \%$ \\
\hline & Median & $0.007 \%$ & $-0.021 \%$ & $0.061 \%$ & $0.076 \%$ & $0.138 \%$ & $0.212 \%$ & $0.247 \%$ & $0.271 \%$ & $0.224 \%$ & $0.126 \%$ & $0.116 \%$ & $0.037 \%$ & $0.079 \%$ & $-0.004 \%$ \\
\hline & Std deviation & $1.541 \%$ & $1.449 \%$ & $1.526 \%$ & $1.444 \%$ & $1.391 \%$ & $1.597 \%$ & $1.335 \%$ & $1.688 \%$ & $1.766 \%$ & $1.583 \%$ & $1.568 \%$ & $1.351 \%$ & $1.546 \%$ & $1.524 \%$ \\
\hline & Student- $\mathrm{t}$ & $-1.993 *$ & -1.432 & 0.596 & 1.952 & -0.485 & 0.962 & 1.532 & 1.586 & 1.238 & 0.629 & -0.142 & -0.075 & -0.330 & -0.935 \\
\hline & Sign & 0.5 & -2 & 6 & 10.5 & 15.5 & $31^{*}$ & $22.5^{*}$ & $37^{*}$ & $26^{*}$ & 12 & 12 & 3 & 10.5 & -0.5 \\
\hline & $\begin{array}{l}\text { Signed Rank } \\
\text { \% Positive }\end{array}$ & -1252.5 & -746 & 1028.5 & 1655.5 & 900.5 & $2992.5^{*}$ & $3830.5^{*}$ & $3988.5^{*}$ & $2666.5^{*}$ & 1063.5 & 997.5 & 318.5 & 793.5 & -647.5 \\
\hline & Days & $50.39 \%$ & $49.22 \%$ & $52.33 \%$ & $54.26 \%$ & $56.20 \% *$ & $62.02 \% *$ & $58.91 \% *$ & $64.34 \% *$ & $60.08 \% *$ & $54.65 \%$ & $54.65 \%$ & $51.16 \%$ & $54.26 \%$ & $50.00 \%$ \\
\hline \multirow{8}{*}{$\begin{array}{l}1989 \\
\text { to } \\
1998\end{array}$} & \# of Obs. & 120 & 120 & 120 & 120 & 120 & 120 & 120 & 120 & 120 & 120 & 120 & 120 & 120 & 120 \\
\hline & Mean & $-0.058 \%$ & $0.069 \%$ & $0.002 \%$ & $0.043 \%$ & $0.086 \%$ & $0.195 \%$ & $0.216 \%$ & $0.196 \%$ & $0.213 \%$ & $0.073 \%$ & $-0.014 \%$ & $0.084 \%$ & $-0.037 \%$ & $0.090 \%$ \\
\hline & Median & $0.061 \%$ & $0.012 \%$ & $0.070 \%$ & $0.084 \%$ & $0.157 \%$ & $0.254 \%$ & $0.370 \%$ & $0.231 \%$ & $0.244 \%$ & $0.144 \%$ & $0.039 \%$ & $0.109 \%$ & $0.128 \%$ & $0.093 \%$ \\
\hline & Std deviation & $0.926 \%$ & $0.936 \%$ & $1.135 \%$ & $0.922 \%$ & $0.933 \%$ & $0.935 \%$ & $1.131 \%$ & $1.046 \%$ & $0.997 \%$ & $0.991 \%$ & $0.972 \%$ & $0.994 \%$ & $0.957 \%$ & $0.956 \%$ \\
\hline & Student-t & -0.682 & 0.809 & 0.023 & 0.507 & 1.006 & $2.285^{*}$ & $2.093^{*}$ & $2.050 *$ & $2.339 *$ & 0.806 & -0.159 & 0.923 & -0.426 & 1.032 \\
\hline & Sign & 3.5 & 0 & 5 & 7.5 & $11.5^{*}$ & $21^{*}$ & $26.5^{*}$ & $17^{*}$ & $20 *$ & $12^{*}$ & 3 & 7 & 9.5 & 4.5 \\
\hline & $\begin{array}{l}\text { Signed Rank } \\
\text { \% Positive }\end{array}$ & 27 & 146.5 & 258 & 260 & 625 & $1110^{*}$ & $2039 *$ & $983 *$ & $1130 *$ & 686 & 184 & 388 & 501 & 488 \\
\hline & Days & $53.33 \%$ & $50.00 \%$ & $54.17 \%$ & $56.67 \% *$ & $60.00 \% *$ & $67.50 \% *$ & $72.50 \% *$ & $64.17 \% *$ & $66.67 \% *$ & $60.00 \% *$ & $52.50 \%$ & $55.83 \%$ & $58.33 \% *$ & $54.17 \%$ \\
\hline \multirow{8}{*}{$\begin{array}{l}1999 \\
\text { to } \\
2010\end{array}$} & \# of Obs. & 138 & 138 & 138 & 138 & 138 & 138 & 138 & 138 & 138 & 138 & 138 & 138 & 138 & 138 \\
\hline & Mean & $-0.307 \%$ & $-0.302 \%$ & $0.104 \%$ & $0.291 \%$ & $-0.153 \%$ & $0.009 \%$ & $0.050 \%$ & $0.141 \%$ & $0.069 \%$ & $0.052 \%$ & $-0.014 \%$ & $-0.085 \%$ & $-0.027 \%$ & $-0.244 \%$ \\
\hline & Median & $-0.111 \%$ & $-0.085 \%$ & $0.038 \%$ & $0.049 \%$ & $0.091 \%$ & $0.128 \%$ & $-0.043 \%$ & $0.319 \%$ & $0.192 \%$ & $0.017 \%$ & $0.217 \%$ & $-0.057 \%$ & $0.023 \%$ & $-0.094 \%$ \\
\hline & Std deviation & $1.919 \%$ & $1.764 \%$ & $1.800 \%$ & $1.774 \%$ & $1.688 \%$ & $2.002 \%$ & $1.490 \%$ & $2.096 \%$ & $2.231 \%$ & $1.962 \%$ & $1.947 \%$ & $1.597 \%$ & $1.920 \%$ & $1.874 \%$ \\
\hline & Student-t & -1.881 & $-2.008 *$ & 0.677 & $1.928^{*}$ & -1.065 & 0.055 & 0.396 & 0.793 & 0.365 & 0.314 & -0.082 & -0.623 & -0.165 & -1.532 \\
\hline & Sign & -3 & -2 & 1 & 3 & 4 & 10 & -4 & $20 *$ & 6 & 0 & 9 & -4 & 1 & -5 \\
\hline & $\begin{array}{l}\text { Signed Rank } \\
\text { \% Positive }\end{array}$ & -660.5 & -617.5 & 245.5 & 620.5 & -169.5 & 408.5 & 114.5 & $1018.5^{*}$ & 267.5 & -33.5 & 252.5 & -201.5 & 15.5 & -762.5 \\
\hline & Days & $47.83 \%$ & $48.55 \%$ & $50.72 \%$ & $52.17 \%$ & $52.90 \%$ & $57.25 \% *$ & $47.10 \%$ & $64.49 \% *$ & $54.35 \%$ & $50.00 \%$ & $56.52 \%$ & $47.10 \%$ & $50.72 \%$ & $46.38 \%$ \\
\hline \multirow{8}{*}{$\begin{array}{l}2007 \\
\text { to } \\
2010\end{array}$} & \# of Obs. & 42 & 42 & 42 & 42 & 42 & 42 & 42 & 42 & 42 & 42 & 42 & 42 & 42 & 42 \\
\hline & Mean & $-0.362 \%$ & $-0.226 \%$ & $-0.099 \%$ & $0.644 \%$ & $0.029 \%$ & $-0.130 \%$ & $-0.127 \%$ & $0.069 \%$ & $0.135 \%$ & $-0.245 \%$ & $-0.441 \%$ & $-0.164 \%$ & $0.163 \%$ & $-0.105 \%$ \\
\hline & Median & $-0.078 \%$ & $-0.112 \%$ & $-0.011 \%$ & $0.254 \%$ & $0.191 \%$ & $0.112 \%$ & $-0.233 \%$ & $0.305 \%$ & $0.151 \%$ & $-0.151 \%$ & $0.094 \%$ & $0.012 \%$ & $0.252 \%$ & $0.010 \%$ \\
\hline & Std deviation & $2.151 \%$ & $1.533 \%$ & $1.473 \%$ & $2.143 \%$ & $1.433 \%$ & $2.314 \%$ & $1.435 \%$ & $2.204 \%$ & $1.609 \%$ & $1.659 \%$ & $1.959 \%$ & $1.840 \%$ & $1.619 \%$ & $2.050 \%$ \\
\hline & Student-t & -1.092 & -0.957 & -0.434 & 1.947 & 0.133 & -0.364 & -0.575 & 0.202 & 0.544 & -0.957 & -1.460 & -0.576 & 0.654 & -0.331 \\
\hline & Sign & 0 & -3 & 0 & 3 & 3 & 5 & -3 & 7* & 3 & -3 & 1 & 0 & 2 & 0 \\
\hline & $\begin{array}{l}\text { Signed Rank } \\
\text { \% Positive }\end{array}$ & -79.5 & -50.5 & -33.5 & 120.5 & 23.5 & 59.5 & -76.5 & 85.5 & 66.5 & -56.5 & -49.5 & -8.5 & 42.5 & -34.5 \\
\hline & Days & $50.00 \%$ & $42.86 \%$ & $50.00 \%$ & $57.14 \%$ & $57.14 \%$ & $61.90 \%$ & $42.86 \%$ & $66.67 \% *$ & $57.14 \%$ & $42.86 \%$ & $52.38 \%$ & $50.00 \%$ & $54.76 \%$ & $50.00 \%$ \\
\hline
\end{tabular}




\section{Table 5: NASDAQ 100 Daily Rates of Return}

The daily rates of return are shown for the first seven and the last seven trading days of the month. The first seven trading days are depicted as positive numbers where 1 is the first trading day and the last seven trading days are depicted by negative numbers where -1 is the last day. The rates of return are calculated as the log of the ratio of the previous day and the current day. Student-t as well as the non-parametric tests - sign, signed rank and binomial proportions-is also reported. * significance at the $95 \%$ level

\begin{tabular}{|c|c|c|c|c|c|c|c|c|c|c|c|c|c|c|c|}
\hline & Trading Day & -7 & -6 & -5 & -4 & -3 & -2 & -1 & 1 & 2 & 3 & 4 & 5 & 6 & 7 \\
\hline 1989 & \# of Obs. & 258 & 258 & 258 & 258 & 258 & 258 & 258 & 258 & 258 & 258 & 258 & 258 & 258 & 258 \\
\hline to & Mean & $-0.189 \%$ & $-0.118 \%$ & $0.084 \%$ & $0.195 \%$ & $-0.071 \%$ & $0.117 \%$ & $-0.005 \%$ & $-0.025 \%$ & $0.112 \%$ & $0.106 \%$ & $-0.045 \%$ & $0.037 \%$ & $-0.043 \%$ & $-0.086 \%$ \\
\hline \multirow[t]{6}{*}{2010} & Median & $-0.016 \%$ & $0.004 \%$ & $0.032 \%$ & $0.102 \%$ & $0.068 \%$ & $0.222 \%$ & $0.119 \%$ & $0.351 \%$ & $0.178 \%$ & $0.082 \%$ & $0.105 \%$ & $0.085 \%$ & $0.170 \%$ & $0.059 \%$ \\
\hline & Std deviation & $1.797 \%$ & $1.783 \%$ & $1.850 \%$ & $1.782 \%$ & $1.720 \%$ & $1.926 \%$ & $1.631 \%$ & $4.803 \%$ & $2.167 \%$ & $1.924 \%$ & $1.882 \%$ & $1.602 \%$ & $1.905 \%$ & $1.769 \%$ \\
\hline & Student-t & -1.687 & -1.063 & 0.728 & 1.754 & -0.664 & 0.980 & -0.049 & -0.083 & 0.833 & 0.881 & -0.386 & 0.372 & -0.361 & -0.781 \\
\hline & Sign & -1 & 0 & 1.5 & 8.5 & 7 & $29 *$ & 11 & $32.5^{*}$ & $20 *$ & 9 & 10 & 7 & 14 & 2 \\
\hline & Signed Rank & -1123.5 & -575.5 & 1028.5 & 1229.5 & 48.5 & $2765.5^{*}$ & 1050.5 & $3833.5^{*}$ & 1816.5 & 991.5 & 15.5 & 798.5 & 666.5 & -404.5 \\
\hline & $\%$ Positive Days & $49.61 \%$ & $50.00 \%$ & $50.78 \%$ & $53.49 \%$ & $52.71 \%$ & $61.24 \% *$ & $54.26 \%$ & $62.79 \% *$ & $57.75 \% *$ & $53.49 \%$ & $53.88 \%$ & $52.71 \%$ & $55.43 \% *$ & $50.78 \%$ \\
\hline \multirow{8}{*}{$\begin{array}{l}1989 \\
\text { to } \\
1998\end{array}$} & \# of Obs. & 120 & 120 & 120 & 120 & 120 & 120 & 120 & 120 & 120 & 120 & 120 & 120 & 120 & 120 \\
\hline & Mean & $-0.025 \%$ & $0.121 \%$ & $0.029 \%$ & $0.085 \%$ & $0.151 \%$ & $0.250 \%$ & $0.042 \%$ & $-0.236 \%$ & $0.157 \%$ & $0.083 \%$ & $-0.083 \%$ & $0.184 \%$ & $-0.023 \%$ & $0.130 \%$ \\
\hline & Median & $0.011 \%$ & $0.073 \%$ & $0.029 \%$ & $0.130 \%$ & $0.145 \%$ & $0.268 \%$ & $0.269 \%$ & $0.372 \%$ & $0.139 \%$ & $0.265 \%$ & $0.029 \%$ & $0.237 \%$ & $0.296 \%$ & $0.124 \%$ \\
\hline & Std deviation & $1.225 \%$ & $1.347 \%$ & $1.454 \%$ & $1.357 \%$ & $1.330 \%$ & $1.364 \%$ & $1.467 \%$ & $6.584 \%$ & $1.430 \%$ & $1.357 \%$ & $1.425 \%$ & $1.305 \%$ & $1.334 \%$ & $1.336 \%$ \\
\hline & Student-t & -0.226 & 0.982 & 0.222 & 0.689 & 1.243 & $2.010^{*}$ & 0.314 & -0.392 & 1.204 & 0.670 & -0.635 & 1.547 & -0.189 & 1.066 \\
\hline & Sign & 1 & 1 & 0.5 & 6 & 5 & $17^{*}$ & $14 *$ & $15^{*}$ & 10 & 8 & 2 & 6 & 9 & 5 \\
\hline & Signed Rank & 48 & 162 & 250 & 161 & 446 & $958 *$ & 731 & 1043* & 562 & 495 & -168 & 587 & 357 & 432 \\
\hline & \% Positive Days & $50.83 \%$ & $50.83 \%$ & $50.83 \%$ & $55.00 \%$ & $54.17 \%$ & $64.17 \% *$ & $61.67 \% *$ & $62.50 \% *$ & $58.33 \% *$ & $56.67 \%$ & $51.67 \%$ & $55.00 \%$ & $57.50 \%$ & $54.17 \%$ \\
\hline \multirow{8}{*}{$\begin{array}{l}1999 \\
\text { to } \\
2010\end{array}$} & \# of Obs. & 137 & 138 & 138 & 138 & 138 & 138 & 138 & 138 & 138 & 138 & 138 & 138 & 138 & 138 \\
\hline & Mean & $0.021 \%$ & $-0.331 \%$ & $-0.326 \%$ & $0.131 \%$ & $0.290 \%$ & $-0.264 \%$ & $0.002 \%$ & $-0.046 \%$ & $0.158 \%$ & $0.074 \%$ & $0.125 \%$ & $-0.013 \%$ & $-0.091 \%$ & $-0.060 \%$ \\
\hline & Median & $0.102 \%$ & $-0.096 \%$ & $-0.037 \%$ & $0.032 \%$ & $0.044 \%$ & $0.055 \%$ & $0.165 \%$ & $-0.077 \%$ & $0.323 \%$ & $0.190 \%$ & $0.008 \%$ & $0.164 \%$ & $0.023 \%$ & $0.122 \%$ \\
\hline & Std deviation & $2.160 \%$ & $2.170 \%$ & $2.072 \%$ & $2.140 \%$ & $2.083 \%$ & $1.982 \%$ & $2.305 \%$ & $1.765 \%$ & $2.357 \%$ & $2.652 \%$ & $2.311 \%$ & $2.209 \%$ & $1.817 \%$ & $2.294 \%$ \\
\hline & Student- $\mathrm{t}$ & 0.112 & -1.791 & -1.846 & 0.720 & 1.633 & -1.565 & 0.011 & -0.305 & 0.789 & 0.326 & 0.636 & -0.068 & -0.588 & -0.307 \\
\hline & Sign & -2 & -1 & 1 & 2.5 & 2 & $12 *$ & -3 & $17.5^{*}$ & 10 & 1 & 8 & 1 & 5 & -3 \\
\hline & Signed Rank & -636.5 & -514.5 & 291.5 & 456.5 & -450.5 & 436.5 & -180.5 & 899.5 & 336.5 & 32.5 & 183.5 & -182.5 & -5.5 & -643.5 \\
\hline & $\%$ Positive Days & $48.55 \%$ & $49.28 \%$ & $50.72 \%$ & $52.17 \%$ & $51.45 \%$ & $58.70 \% *$ & $47.83 \%$ & $63.04 \% *$ & $57.25 \% *$ & $50.72 \%$ & $55.80 \%$ & $50.72 \%$ & $53.62 \%$ & $47.83 \%$ \\
\hline \multirow{8}{*}{$\begin{array}{l}2007 \\
\text { to } \\
2010\end{array}$} & $\#$ of Obs. & 42 & 42 & 42 & 42 & 42 & 42 & 42 & 42 & 42 & 42 & 42 & 42 & 42 & 42 \\
\hline & Mean & $-0.342 \%$ & $-0.130 \%$ & $-0.072 \%$ & $0.693 \%$ & $-0.013 \%$ & $-0.141 \%$ & $-0.212 \%$ & $0.090 \%$ & $0.155 \%$ & $-0.140 \%$ & $-0.407 \%$ & $-0.154 \%$ & $0.206 \%$ & $-0.096 \%$ \\
\hline & Median & $-0.281 \%$ & $-0.152 \%$ & $0.055 \%$ & $0.137 \%$ & $0.188 \%$ & $0.217 \%$ & $-0.329 \%$ & $0.220 \%$ & $0.195 \%$ & $-0.004 \%$ & $0.003 \%$ & $0.070 \%$ & $0.236 \%$ & $0.089 \%$ \\
\hline & Std deviation & $2.061 \%$ & $1.518 \%$ & $1.440 \%$ & $2.276 \%$ & $1.470 \%$ & $2.449 \%$ & $1.552 \%$ & $2.144 \%$ & $1.640 \%$ & $1.755 \%$ & $1.914 \%$ & $1.848 \%$ & $1.634 \%$ & $1.925 \%$ \\
\hline & Student-t & -1.076 & -0.555 & -0.324 & $1.973^{*}$ & -0.057 & -0.372 & -0.884 & 0.272 & 0.613 & -0.516 & -1.379 & -0.540 & 0.817 & -0.323 \\
\hline & Sign & -1 & -2 & 1 & 3 & 3 & 9* & -2 & 5 & 5 & 0 & 1 & 3 & 4 & 1 \\
\hline & Signed Rank & -68.5 & -19.5 & -0.5 & 121.5 & -6.5 & 102.5 & -111.5 & 74.5 & 88.5 & -5.5 & -59.5 & 2.5 & 71.5 & -22.5 \\
\hline & \% Positive Days & $47.62 \%$ & $45.24 \%$ & $52.38 \%$ & $57.14 \%$ & $57.14 \%$ & $71.43 \% *$ & $45.24 \%$ & $61.90 \%$ & $61.90 \%$ & $50.00 \%$ & $52.38 \%$ & $57.14 \%$ & $59.52 \%$ & $52.38 \%$ \\
\hline
\end{tabular}




\section{Table 6: S\&P 500 Daily Rates of Return}

The daily rates of return are shown for the first seven and the last seven trading days of the month. The first seven trading days are depicted as positive numbers where 1 is the first trading day and the last seven trading days are depicted by negative numbers where -1 is the last day. The rates of return are calculated as the log of the ratio of the previous day and the current day. Student-t as well as the non-parametric tests - sign, signed rank and binomial proportions- is also reported. $*$ - significance at the $95 \%$ level

\begin{tabular}{|c|c|c|c|c|c|c|c|c|c|c|c|c|c|c|c|}
\hline & Trading Day & -7 & -6 & -5 & -4 & -3 & -2 & -1 & 1 & 2 & 3 & 4 & 5 & 6 & 7 \\
\hline 1989 & \# of Obs. & 258 & 258 & 258 & 258 & 258 & 258 & 258 & 258 & 258 & 258 & 258 & 258 & 258 & 258 \\
\hline to & Mean & $-0.150 \%$ & $-0.081 \%$ & $0.007 \%$ & $0.154 \%$ & $0.048 \%$ & $0.082 \%$ & $0.014 \%$ & $0.235 \%$ & $0.089 \%$ & $-0.036 \%$ & $-0.048 \%$ & $-0.019 \%$ & $-0.020 \%$ & $-0.109 \%$ \\
\hline \multirow[t]{6}{*}{2010} & Median & $-0.055 \%$ & $-0.018 \%$ & $0.030 \%$ & $0.012 \%$ & $0.105 \%$ & $0.120 \%$ & $0.002 \%$ & $0.265 \%$ & $0.080 \%$ & $0.001 \%$ & $-0.004 \%$ & $-0.022 \%$ & $0.048 \%$ & $-0.003 \%$ \\
\hline & Std deviation & $1.200 \%$ & $1.163 \%$ & $1.105 \%$ & $1.231 \%$ & $1.011 \%$ & $1.272 \%$ & $1.048 \%$ & $1.281 \%$ & $1.153 \%$ & $1.051 \%$ & $1.196 \%$ & $1.077 \%$ & $1.059 \%$ & $1.225 \%$ \\
\hline & Student-t & $-2.014^{*}$ & -1.122 & 0.103 & $2.007^{*}$ & 0.769 & 1.036 & 0.215 & $2.943 *$ & 1.235 & -0.551 & -0.638 & -0.286 & -0.306 & -1.433 \\
\hline & Sign & -5 & -1 & 4 & 3.5 & $17^{*}$ & $25 *$ & 2 & $30 *$ & $19.5^{*}$ & 0.5 & -1 & -4 & 2 & -1.5 \\
\hline & Signed Rank & -1917.5 & -1061.5 & 773.5 & 1467.5 & 1647.5 & $2688.5^{*}$ & 788.5 & $5608.5^{*}$ & $2137.5^{*}$ & -700.5 & 175.5 & -274.5 & -101.5 & -1171.5 \\
\hline & $\%$ Positive Days & $48.06 \%$ & $49.61 \%$ & $51.55 \%$ & $51.55 \%$ & $56.59 \%$ & $59.69 \%$ & $50.78 \%$ & $61.63 \% *$ & $57.75 \% *$ & $50.39 \%$ & $49.61 \%$ & $48.45 \%$ & $50.78 \%$ & $49.61 \%$ \\
\hline \multirow{8}{*}{$\begin{array}{l}1989 \\
\text { to } \\
1998\end{array}$} & \# of Obs. & 120 & 120 & 120 & 120 & 120 & 120 & 120 & 120 & 120 & 120 & 120 & 120 & 120 & 120 \\
\hline & Mean & $-0.098 \%$ & $0.084 \%$ & $-0.007 \%$ & $0.068 \%$ & $0.103 \%$ & $0.136 \%$ & $0.016 \%$ & $0.281 \%$ & $0.152 \%$ & $-0.037 \%$ & $-0.042 \%$ & $0.069 \%$ & $-0.054 \%$ & $0.063 \%$ \\
\hline & Median & $-0.025 \%$ & $-0.041 \%$ & $0.137 \%$ & $0.015 \%$ & $0.149 \%$ & $0.174 \%$ & $0.085 \%$ & $0.247 \%$ & $0.090 \%$ & $0.002 \%$ & $-0.098 \%$ & $0.037 \%$ & $-0.005 \%$ & $0.036 \%$ \\
\hline & Std deviation & $0.761 \%$ & $0.870 \%$ & $1.001 \%$ & $0.828 \%$ & $0.781 \%$ & $0.825 \%$ & $1.011 \%$ & $0.903 \%$ & $0.860 \%$ & $0.723 \%$ & $0.797 \%$ & $0.872 \%$ & $0.788 \%$ & $0.813 \%$ \\
\hline & Student- $\mathrm{t}$ & -1.404 & 1.062 & -0.078 & 0.897 & 1.448 & 1.811 & 0.169 & $3.408^{*}$ & 1.930 & -0.557 & -0.577 & 0.863 & -0.757 & 0.853 \\
\hline & Sign & -1 & -1 & 7 & 1.5 & $13^{*}$ & $16^{*}$ & 7 & $12^{*}$ & $12 *$ & 0.5 & -11 & 1 & 0 & 5 \\
\hline & Signed Rank & -308 & 160 & 408 & 367 & $806^{*}$ & $869 *$ & 492 & $1273 *$ & $829 *$ & -208 & -279 & 189 & -55 & 428 \\
\hline & $\%$ Positive Days & $49.17 \%$ & $49.17 \%$ & $55.83 \%$ & $51.67 \%$ & $60.83 \%$ & $63.33 \%$ & $55.83 \%$ & $60.00 \% *$ & $60.00 \%$ & $50.83 \%$ & $40.83 \%$ & $50.83 \%$ & $50.00 \%$ & $54.17 \%$ \\
\hline \multirow{8}{*}{$\begin{array}{l}1999 \\
\text { to } \\
2010\end{array}$} & $\#$ of Obs. & 138 & 138 & 138 & 138 & 138 & 138 & 138 & 138 & 138 & 138 & 138 & 138 & 138 & 138 \\
\hline & Mean & $-0.196 \%$ & $-0.225 \%$ & $0.019 \%$ & $0.229 \%$ & $0.001 \%$ & $0.035 \%$ & $0.013 \%$ & $0.195 \%$ & $0.034 \%$ & $-0.036 \%$ & $-0.052 \%$ & $-0.096 \%$ & $0.010 \%$ & $-0.259 \%$ \\
\hline & Median & $-0.120 \%$ & $-0.004 \%$ & $-0.055 \%$ & $0.012 \%$ & $0.053 \%$ & $0.076 \%$ & $-0.068 \%$ & $0.378 \%$ & $0.073 \%$ & $-0.016 \%$ & $0.176 \%$ & $-0.106 \%$ & $0.064 \%$ & $-0.072 \%$ \\
\hline & Std deviation & $1.481 \%$ & $1.354 \%$ & $1.192 \%$ & $1.496 \%$ & $1.176 \%$ & $1.561 \%$ & $1.083 \%$ & $1.539 \%$ & $1.358 \%$ & $1.273 \%$ & $1.460 \%$ & $1.226 \%$ & $1.250 \%$ & $1.480 \%$ \\
\hline & Student-t & -1.559 & -1.954 & 0.192 & 1.796 & 0.008 & 0.261 & 0.138 & 1.486 & 0.294 & -0.328 & -0.421 & -0.916 & 0.091 & $-2.059 *$ \\
\hline & Sign & -4 & 0 & -3 & 2 & 4 & 9 & -5 & $18^{*}$ & 7.5 & 0 & 10 & -5 & 2 & -6.5 \\
\hline & Signed Rank & -656.5 & -701.5 & -6.5 & 457.5 & 36.5 & 503.5 & -77.5 & $1508.5^{*}$ & 227.5 & -132.5 & 213.5 & -322.5 & -9.5 & $-1026.5^{*}$ \\
\hline & $\%$ Positive Days & $47.10 \%$ & $50.00 \%$ & $47.83 \%$ & $51.45 \%$ & $52.90 \%$ & $56.52 \%$ & $46.38 \%$ & $63.04 \%$ & $55.80 \%$ & $50.00 \%$ & $57.25 \%$ & $46.38 \%$ & $51.45 \%$ & $45.65 \%$ \\
\hline \multirow{8}{*}{$\begin{array}{l}2007 \\
\text { to } \\
2010\end{array}$} & $\#$ of Obs. & 42 & 42 & 42 & 42 & 42 & 42 & 42 & 42 & 42 & 42 & 42 & 42 & 42 & 42 \\
\hline & Mean & $-0.265 \%$ & $-0.343 \%$ & $-0.169 \%$ & $0.608 \%$ & $0.041 \%$ & $-0.097 \%$ & $-0.139 \%$ & $0.037 \%$ & $0.128 \%$ & $-0.253 \%$ & $-0.419 \%$ & $-0.159 \%$ & $0.225 \%$ & $-0.292 \%$ \\
\hline & Median & $0.096 \%$ & $-0.025 \%$ & $-0.101 \%$ & $0.098 \%$ & $-0.085 \%$ & $0.012 \%$ & $-0.137 \%$ & $0.409 \%$ & $0.101 \%$ & $0.001 \%$ & $0.218 \%$ & $0.107 \%$ & $0.161 \%$ & $0.036 \%$ \\
\hline & Std deviation & $2.084 \%$ & $1.595 \%$ & $1.517 \%$ & $2.216 \%$ & $1.284 \%$ & $2.201 \%$ & $1.443 \%$ & $2.152 \%$ & $1.596 \%$ & $1.528 \%$ & $1.922 \%$ & $1.715 \%$ & $1.499 \%$ & $2.211 \%$ \\
\hline & Student-t & -0.824 & -1.394 & -0.721 & 1.777 & 0.206 & -0.284 & -0.622 & 0.112 & 0.519 & -1.073 & -1.412 & -0.602 & 0.975 & -0.856 \\
\hline & Sign & 0 & 0 & -3 & 4 & -1 & 1 & -2 & 5 & 2.5 & 0 & 4 & 2 & 2 & 1 \\
\hline & Signed Rank & -65.5 & -64.5 & -70.5 & 117.5 & 22.5 & 29.5 & -71.5 & 106.5 & 18.5 & -64.5 & -52.5 & 4.5 & 75.5 & -58.5 \\
\hline & \% Positive Days & $50.00 \%$ & $50.00 \%$ & $42.86 \%$ & $59.52 \%$ & $47.62 \%$ & $52.38 \%$ & $45.24 \%$ & $61.90 \%$ & $57.14 \%$ & $50.00 \%$ & $59.52 \%$ & $54.76 \%$ & $54.76 \%$ & $52.38 \%$ \\
\hline
\end{tabular}




\section{Table 7: Russell 3000 Daily Rates of Return}

The daily rates of return are shown for the first seven and the last seven trading days of the month. The first seven trading days are depicted as positive numbers where 1 is the first trading day and the last seven trading days are depicted by negative numbers where -1 is the last day. The rates of return are calculated as the log of the ratio of the previous day and the current day. Student-t as well as the non-parametric tests - sign, signed rank and binomial proportions- is also reported. * - significance at the $95 \%$ level

\begin{tabular}{|c|c|c|c|c|c|c|c|c|c|c|c|c|c|c|c|}
\hline & Trading Day & -7 & -6 & -5 & -4 & -3 & -2 & -1 & 1 & 2 & 3 & 4 & 5 & 6 & 7 \\
\hline \multirow{8}{*}{$\begin{array}{l}1989 \\
\text { to } \\
2010\end{array}$} & \# of Obs. & 258 & 258 & 258 & 258 & 258 & 258 & 258 & 258 & 258 & 258 & 258 & 258 & 258 & 258 \\
\hline & Mean & $-0.156 \%$ & $-0.090 \%$ & $0.004 \%$ & $0.157 \%$ & $0.050 \%$ & $0.107 \%$ & $0.061 \%$ & $0.211 \%$ & $0.102 \%$ & $-0.030 \%$ & $-0.044 \%$ & $-0.030 \%$ & $-0.028 \%$ & $-0.109 \%$ \\
\hline & Median & $-0.023 \%$ & $-0.039 \%$ & $0.021 \%$ & $0.048 \%$ & $0.129 \%$ & $0.152 \%$ & $0.059 \%$ & $0.285 \%$ & $0.138 \%$ & $0.027 \%$ & $0.006 \%$ & $-0.009 \%$ & $0.025 \%$ & $0.001 \%$ \\
\hline & Std deviation & $1.203 \%$ & $1.144 \%$ & $1.090 \%$ & $1.206 \%$ & $1.007 \%$ & $1.260 \%$ & $1.012 \%$ & $1.290 \%$ & $1.165 \%$ & $1.062 \%$ & $1.199 \%$ & $1.072 \%$ & $1.062 \%$ & $1.222 \%$ \\
\hline & Student-t & $-2.086^{*}$ & -1.266 & 0.059 & $2.091^{*}$ & 0.801 & 1.369 & 0.967 & $2.625^{*}$ & 1.408 & -0.450 & -0.594 & -0.450 & -0.430 & -1.428 \\
\hline & Sign & -5 & -5 & 3 & 7 & $22.5^{*}$ & $25^{*}$ & 10.5 & $32 *$ & 16 & 3 & 1 & -2 & 7.5 & 0 \\
\hline & Signed Rank & -1788.5 & -1096.5 & 611.5 & 1708.5 & 1839 & 3312.5 & 2063.5 & $5406.5^{*}$ & $2507^{*}$ & -460.5 & 456.5 & -329.5 & -129 & -1135.5 \\
\hline & \% Positive Days & $48.06 \%$ & $48.06 \%$ & $51.16 \%$ & $52.71 \%$ & $59.30 \%$ & $59.69 \%$ & $54.26 \%$ & $62.40 \% *$ & $56.59 \% *$ & $51.16 \%$ & $50.39 \%$ & $49.22 \%$ & $53.49 \%$ & $50.00 \%$ \\
\hline \multirow{8}{*}{$\begin{array}{l}1989 \\
\text { to } \\
1998\end{array}$} & \# of Obs. & 120 & 120 & 120 & 120 & 120 & 120 & 120 & 120 & 120 & 120 & 120 & 120 & 120 & 120 \\
\hline & Mean & $-0.096 \%$ & $0.066 \%$ & $-0.012 \%$ & $0.058 \%$ & $0.103 \%$ & $0.157 \%$ & $0.072 \%$ & $0.254 \%$ & $0.176 \%$ & $-0.019 \%$ & $-0.033 \%$ & $0.050 \%$ & $-0.059 \%$ & $0.055 \%$ \\
\hline & Median & $-0.020 \%$ & $-0.039 \%$ & $0.057 \%$ & $0.073 \%$ & $0.160 \%$ & $0.216 \%$ & $0.129 \%$ & $0.216 \%$ & $0.140 \%$ & $0.052 \%$ & $-0.074 \%$ & $0.028 \%$ & $0.026 \%$ & $0.059 \%$ \\
\hline & Std deviation & $0.729 \%$ & $0.811 \%$ & $0.951 \%$ & $0.771 \%$ & $0.747 \%$ & $0.778 \%$ & $0.950 \%$ & $0.852 \%$ & $0.832 \%$ & $0.702 \%$ & $0.769 \%$ & $0.834 \%$ & $0.756 \%$ & $0.773 \%$ \\
\hline & Student-t & -1.446 & 0.895 & -0.133 & 0.817 & 1.511 & $2.205^{*}$ & 0.824 & $3.269 *$ & $2.323^{*}$ & -0.290 & -0.474 & 0.655 & -0.860 & 0.779 \\
\hline & Sign & -3 & -3 & 5 & 5 & $17.5^{*}$ & $14 *$ & 10.5 & $15^{*}$ & 10.5 & 6 & -7 & 1 & 5 & 7 \\
\hline & Signed Rank & -272 & 86 & 400 & 406 & 918* & $1091^{*}$ & $981^{*}$ & $1254^{*}$ & $1015^{*}$ & -34 & -183 & 154 & 33.5 & 451 \\
\hline & $\%$ Positive I & $47.50 \%$ & $47.50 \%$ & $54.17 \%$ & $54.17 \%$ & $65.00 \% *$ & $61.67 \% *$ & $59.17 \% *$ & $62.50 \% *$ & $59.17 \% *$ & $55.00 \%$ & $44.17 \%$ & $50.83 \%$ & $55.00 \%$ & $55.83 \%$ \\
\hline \multirow{8}{*}{$\begin{array}{l}1999 \\
\text { to } \\
2010\end{array}$} & \# of Obs. & 138 & 138 & 138 & 138 & 138 & 138 & 138 & 138 & 138 & 138 & 138 & 138 & 138 & 138 \\
\hline & Mean & $-0.208 \%$ & $-0.226 \%$ & $0.017 \%$ & $0.244 \%$ & $0.004 \%$ & $0.065 \%$ & $0.052 \%$ & $0.173 \%$ & $0.037 \%$ & $-0.039 \%$ & $-0.054 \%$ & $-0.100 \%$ & $-0.002 \%$ & $-0.251 \%$ \\
\hline & Median & $-0.048 \%$ & $-0.047 \%$ & $-0.030 \%$ & $0.027 \%$ & $0.064 \%$ & $0.097 \%$ & $0.000 \%$ & $0.362 \%$ & $0.095 \%$ & $-0.042 \%$ & $0.164 \%$ & $-0.041 \%$ & $0.025 \%$ & $-0.077 \%$ \\
\hline & Std deviation & $1.499 \%$ & $1.358 \%$ & $1.201 \%$ & $1.482 \%$ & $1.189 \%$ & $1.564 \%$ & $1.067 \%$ & $1.577 \%$ & $1.391 \%$ & $1.299 \%$ & $1.478 \%$ & $1.241 \%$ & $1.273 \%$ & $1.496 \%$ \\
\hline & Student- $\mathrm{t}$ & -1.633 & -1.956 & 0.171 & 1.930 & 0.042 & 0.484 & 0.571 & 1.288 & 0.316 & -0.357 & -0.429 & -0.943 & -0.014 & -1.970 \\
\hline & Sign & -2 & -2 & -2 & 2 & 5 & 11 & 0 & $17^{*}$ & 5.5 & -3 & 8 & -3 & 2.5 & -7 \\
\hline & Signed Rank & -648.5 & -687.5 & -26.5 & 534.5 & 39 & 623.5 & 162.5 & $1423.5^{*}$ & 234.5 & -173.5 & 263.5 & -333.5 & -65.5 & $-994.5^{*}$ \\
\hline & \% Positive Days & $48.55 \%$ & $48.55 \%$ & $48.55 \%$ & $51.45 \%$ & $54.35 \%$ & $57.97 \% *$ & $50.00 \%$ & $62.32 \% *$ & $54.35 \%$ & $47.83 \%$ & $55.80 \%$ & $47.83 \%$ & $52.17 \%$ & $44.93 \%$ \\
\hline \multirow{8}{*}{$\begin{array}{l}2007 \\
\text { to } \\
2010\end{array}$} & \# of Obs. & 42 & 42 & 42 & 42 & 42 & 42 & 42 & 42 & 42 & 42 & 42 & 42 & 42 & 42 \\
\hline & Mean & $-0.292 \%$ & $-0.353 \%$ & $-0.184 \%$ & $0.614 \%$ & $0.057 \%$ & $-0.081 \%$ & $-0.122 \%$ & $0.031 \%$ & $0.128 \%$ & $-0.270 \%$ & $-0.436 \%$ & $-0.156 \%$ & $0.221 \%$ & $-0.267 \%$ \\
\hline & Median & $0.112 \%$ & $-0.095 \%$ & $-0.138 \%$ & $0.078 \%$ & $0.000 \%$ & $0.014 \%$ & $-0.136 \%$ & $0.420 \%$ & $0.179 \%$ & $-0.070 \%$ & $0.219 \%$ & $0.113 \%$ & $0.102 \%$ & $0.006 \%$ \\
\hline & Std deviation & $2.126 \%$ & $1.618 \%$ & $1.527 \%$ & $2.191 \%$ & $1.308 \%$ & $2.217 \%$ & $1.420 \%$ & $2.221 \%$ & $1.656 \%$ & $1.550 \%$ & $1.955 \%$ & $1.745 \%$ & $1.542 \%$ & $2.230 \%$ \\
\hline & Student-t & -0.891 & -1.414 & -0.780 & 1.817 & 0.281 & -0.237 & -0.556 & 0.092 & 0.499 & -1.130 & -1.444 & -0.580 & 0.928 & -0.775 \\
\hline & Sign & 1 & -1 & -3 & 4 & 0 & 1 & -3 & 6 & 3 & -1 & 4 & 3 & 3 & 0 \\
\hline & Signed Rank & -61.5 & -55.5 & -82.5 & 123.5 & 26 & 26.5 & -65.5 & 109.5 & 33.5 & -78.5 & -51.5 & -3.5 & 71.5 & -46.5 \\
\hline & \% Positive Days & $52.38 \%$ & $47.62 \%$ & $42.86 \%$ & $59.52 \%$ & $52.38 \%$ & $52.38 \%$ & $42.86 \%$ & $64.29 \% *$ & $57.14 \%$ & $47.62 \%$ & $59.52 \%$ & $57.14 \%$ & $57.14 \%$ & $50.00 \%$ \\
\hline
\end{tabular}




\section{Table 8: Russell 2000 Daily Rates of Return}

The daily rates of return are shown for the first seven and the last seven trading days of the month. The first seven trading days are depicted as positive numbers where 1 is the first trading day and the last seven trading days are depicted by negative numbers where -1 is the last day. The rates of return are calculated as the log of the ratio of the previous day and the current day. Student-t as well as the non-parametric tests - sign, signed rank and binomial proportions-is also reported. $*$ - significance at the $95 \%$ level

\begin{tabular}{|c|c|c|c|c|c|c|c|c|c|c|c|c|c|c|c|}
\hline & Trading Day & -7 & -6 & -5 & -4 & -3 & -2 & -1 & 1 & 2 & 3 & 4 & 5 & 6 & 7 \\
\hline \multirow{8}{*}{$\begin{array}{l}1989 \\
\text { to } \\
2010\end{array}$} & $\#$ of Obs. & 258 & 258 & 258 & 258 & 258 & 258 & 258 & 258 & 258 & 258 & 258 & 258 & 258 & 258 \\
\hline & Mean & $-0.191 \%$ & $-0.106 \%$ & $-0.006 \%$ & $0.195 \%$ & $0.055 \%$ & $0.166 \%$ & $0.257 \%$ & $0.099 \%$ & $0.152 \%$ & $-0.064 \%$ & $0.001 \%$ & $-0.059 \%$ & $-0.057 \%$ & $-0.082 \%$ \\
\hline & Median & $-0.008 \%$ & $-0.035 \%$ & $0.046 \%$ & $0.146 \%$ & $0.194 \%$ & $0.224 \%$ & $0.321 \%$ & $0.230 \%$ & $0.191 \%$ & $0.013 \%$ & $0.148 \%$ & $0.014 \%$ & $0.039 \%$ & $0.052 \%$ \\
\hline & Std deviation & $1.426 \%$ & $1.187 \%$ & $1.207 \%$ & $1.289 \%$ & $1.201 \%$ & $1.362 \%$ & $1.116 \%$ & $1.526 \%$ & $1.322 \%$ & $1.218 \%$ & $1.313 \%$ & $1.240 \%$ & $1.259 \%$ & $1.380 \%$ \\
\hline & Student-t & $-2.151^{*}$ & -1.428 & -0.080 & $2.428^{*}$ & 0.734 & $1.956^{*}$ & $3.704 *$ & 1.043 & 1.851 & -0.844 & 0.014 & -0.767 & -0.728 & -0.959 \\
\hline & Sign & -0.5 & -5 & 11 & $17^{*}$ & $23^{*}$ & $32 *$ & $46^{*}$ & $27 *$ & $32 *$ & 5 & $21 *$ & 7.5 & 6 & 7.5 \\
\hline & $\begin{array}{l}\text { Signed Rank } \\
\text { \% Positive }\end{array}$ & -1656.5 & -894.5 & 540.5 & $2870.5^{*}$ & $2105.5^{*}$ & $3855.5^{*}$ & $5695.5^{*}$ & $3684.5^{*}$ & $3483.5^{*}$ & -440.5 & 1810.5 & -313.5 & -3.5 & -845.5 \\
\hline & Days & $50.00 \%$ & $48.06 \%$ & $54.26 \%$ & $56.59 \% *$ & $58.91 \% *$ & $62.40 \% *$ & $67.83 \% *$ & $60.47 \% *$ & $62.40 \% *$ & $51.94 \%$ & $58.14 \% *$ & $53.10 \%$ & $52.33 \%$ & $53.10 \%$ \\
\hline \multirow{8}{*}{$\begin{array}{l}1989 \\
\text { to } \\
1998\end{array}$} & \# of Obs. & 120 & 120 & 120 & 120 & 120 & 120 & 120 & 120 & 120 & 120 & 120 & 120 & 120 & 120 \\
\hline & Mean & $-0.083 \%$ & $0.015 \%$ & $-0.037 \%$ & $-0.012 \%$ & $0.038 \%$ & $0.177 \%$ & $0.386 \%$ & $0.097 \%$ & $0.231 \%$ & $0.028 \%$ & $0.036 \%$ & $0.009 \%$ & $-0.068 \%$ & $0.034 \%$ \\
\hline & Median & $0.025 \%$ & $0.042 \%$ & $0.062 \%$ & $0.092 \%$ & $0.162 \%$ & $0.256 \%$ & $0.478 \%$ & $0.197 \%$ & $0.211 \%$ & $0.093 \%$ & $0.102 \%$ & $0.054 \%$ & $0.091 \%$ & $0.091 \%$ \\
\hline & Std deviation & $0.809 \%$ & $0.661 \%$ & $0.908 \%$ & $0.687 \%$ & $0.730 \%$ & $0.683 \%$ & $0.836 \%$ & $0.736 \%$ & $0.748 \%$ & $0.771 \%$ & $0.699 \%$ & $0.789 \%$ & $0.791 \%$ & $0.689 \%$ \\
\hline & Student-t & -1.124 & 0.255 & -0.441 & -0.199 & 0.565 & $2.834^{*}$ & $5.058 *$ & 1.437 & $3.382 *$ & 0.395 & 0.567 & 0.132 & -0.938 & 0.539 \\
\hline & Sign & 1.5 & 1 & 9 & 6 & $12^{*}$ & $25^{*}$ & $42 *$ & $14^{*}$ & $27^{*}$ & 10 & 11 & 8.5 & 11 & 11 \\
\hline & $\begin{array}{l}\text { Signed Rank } \\
\% \text { Positive }\end{array}$ & -51 & 234 & 332 & 265 & 590 & $1483^{*}$ & $2684^{*}$ & $844^{*}$ & $1625^{*}$ & 498 & 718 & 122 & 420 & 424 \\
\hline & Days & $51.67 \%$ & $50.83 \%$ & $57.50 \% *$ & $55.00 \%$ & $60.00 \% *$ & $70.83 \% *$ & $85.00 \% *$ & $61.67 \% *$ & $72.50 \% *$ & $58.33 \% *$ & $59.17 \% *$ & $57.50 \% *$ & $59.17 \% *$ & $59.17 \% *$ \\
\hline \multirow{8}{*}{$\begin{array}{l}1999 \\
\text { to } \\
2010\end{array}$} & \# of Obs. & 138 & 138 & 138 & 138 & 138 & 138 & 138 & 138 & 138 & 138 & 138 & 138 & 138 & 138 \\
\hline & Mean & $-0.285 \%$ & $-0.211 \%$ & $0.021 \%$ & $0.375 \%$ & $0.070 \%$ & $0.156 \%$ & $0.146 \%$ & $0.101 \%$ & $0.084 \%$ & $-0.144 \%$ & $-0.029 \%$ & $-0.119 \%$ & $-0.048 \%$ & $-0.184 \%$ \\
\hline & Median & $-0.116 \%$ & $-0.115 \%$ & $0.021 \%$ & $0.300 \%$ & $0.228 \%$ & $0.143 \%$ & $0.094 \%$ & $0.285 \%$ & $0.159 \%$ & $-0.146 \%$ & $0.267 \%$ & $-0.021 \%$ & $-0.093 \%$ & $-0.208 \%$ \\
\hline & Std deviation & $1.796 \%$ & $1.497 \%$ & $1.419 \%$ & $1.624 \%$ & $1.497 \%$ & $1.754 \%$ & $1.305 \%$ & $1.974 \%$ & $1.668 \%$ & $1.502 \%$ & $1.675 \%$ & $1.528 \%$ & $1.560 \%$ & $1.771 \%$ \\
\hline & Student-t & -1.863 & -1.653 & 0.171 & $2.713^{*}$ & 0.548 & 1.048 & 1.311 & 0.602 & 0.592 & -1.125 & -0.205 & -0.915 & -0.361 & -1.218 \\
\hline & Sign & -2 & -6 & 2 & 11 & 11 & 7 & 4 & $13^{*}$ & 5 & -5 & 10 & -1 & -5 & -3.5 \\
\hline & $\begin{array}{l}\text { Signed Rank } \\
\text { \% Positive }\end{array}$ & -797.5 & -638.5 & 53.5 & $1137.5^{*}$ & 426.5 & 616.5 & 407.5 & $981.5^{*}$ & 354.5 & -578.5 & 243.5 & -288.5 & -185.5 & -667.5 \\
\hline & Days & $48.55 \%$ & $45.65 \%$ & $51.45 \%$ & 57.97\%* & $57.97 \% *$ & $55.07 \%$ & $52.90 \%$ & $59.42 \% *$ & $53.62 \%$ & $46.38 \%$ & $57.25 \% *$ & $49.28 \%$ & $46.38 \%$ & $47.83 \%$ \\
\hline \multirow{8}{*}{$\begin{array}{l}2007 \\
\text { to } \\
2010\end{array}$} & \# of Obs. & 42 & 42 & 42 & 42 & 42 & 42 & 42 & 42 & 42 & 42 & 42 & 42 & 42 & 42 \\
\hline & Mean & $-0.410 \%$ & $-0.453 \%$ & $-0.253 \%$ & $0.720 \%$ & $0.081 \%$ & $-0.057 \%$ & $-0.158 \%$ & $-0.001 \%$ & $0.147 \%$ & $-0.458 \%$ & $-0.453 \%$ & $-0.156 \%$ & $0.132 \%$ & $-0.135 \%$ \\
\hline & Median & $-0.013 \%$ & $-0.195 \%$ & $-0.279 \%$ & $0.372 \%$ & $0.303 \%$ & $-0.018 \%$ & $-0.531 \%$ & $0.296 \%$ & $0.274 \%$ & $-0.446 \%$ & $0.286 \%$ & $-0.052 \%$ & $-0.031 \%$ & $0.109 \%$ \\
\hline & Std deviation & $2.651 \%$ & $1.817 \%$ & $1.802 \%$ & $2.251 \%$ & $1.746 \%$ & $2.464 \%$ & $1.639 \%$ & $2.784 \%$ & $2.072 \%$ & $1.809 \%$ & $2.282 \%$ & $2.148 \%$ & $2.021 \%$ & $2.596 \%$ \\
\hline & Student-t & -1.002 & -1.618 & -0.910 & $2.074^{*}$ & 0.302 & -0.151 & -0.624 & -0.002 & 0.460 & -1.641 & -1.287 & -0.470 & 0.423 & -0.337 \\
\hline & Sign & 0 & -4 & -2 & 3 & 3 & 0 & -4 & 5 & 3 & -4 & 4 & 0 & 0 & 3 \\
\hline & $\begin{array}{l}\text { Signed Rank } \\
\text { \% Positive }\end{array}$ & -91.5 & -101.5 & -87.5 & 134.5 & 63.5 & -14.5 & -97.5 & 108.5 & 46.5 & -122.5 & -49.5 & -20.5 & 20.5 & -17.5 \\
\hline & Days & $50.00 \%$ & $40.48 \%$ & $45.24 \%$ & $57.14 \%$ & $57.14 \%$ & $50.00 \%$ & $40.48 \%$ & $61.90 \%$ & $57.14 \%$ & $40.48 \%$ & $59.52 \%$ & $50.00 \%$ & $50.00 \%$ & $57.14 \%$ \\
\hline
\end{tabular}




\section{Table 9: Russell 1000 Daily Rates of Return}

The daily rates of return are shown for the first seven and the last seven trading days of the month. The first seven trading days are depicted as positive numbers where 1 is the first trading day and the last seven trading days are depicted by negative numbers where -1 is the last day. The rates of return are calculated as the log of the ratio of the previous day and the current day. Student-t as well as the non-parametric tests - sign, signed rank and binomial proportions-is also reported. $*$ - significance at the $95 \%$ level

\begin{tabular}{|c|c|c|c|c|c|c|c|c|c|c|c|c|c|c|c|}
\hline & Trading Day & -7 & -6 & -5 & -4 & -3 & -2 & -1 & 1 & 2 & 3 & 4 & 5 & 6 & 7 \\
\hline \multirow{8}{*}{$\begin{array}{l}1989 \\
\text { to } \\
2010\end{array}$} & \# of Obs. & 211 & 211 & 211 & 211 & 211 & 211 & 211 & 210 & 210 & 210 & 210 & 210 & 210 & 210 \\
\hline & Mean & $-0.140 \%$ & $-0.200 \%$ & $0.093 \%$ & $0.164 \%$ & $0.029 \%$ & $0.089 \%$ & $-0.020 \%$ & $0.208 \%$ & $0.084 \%$ & $-0.008 \%$ & $-0.042 \%$ & $-0.027 \%$ & $-0.045 \%$ & $-0.148 \%$ \\
\hline & Median & $-0.020 \%$ & $-0.030 \%$ & $0.035 \%$ & $0.028 \%$ & $0.081 \%$ & $0.124 \%$ & $0.010 \%$ & $0.308 \%$ & $0.110 \%$ & $0.033 \%$ & $0.009 \%$ & $0.007 \%$ & $0.033 \%$ & $0.007 \%$ \\
\hline & Std deviation & $1.263 \%$ & $1.435 \%$ & $1.420 \%$ & $1.300 \%$ & $1.056 \%$ & $1.346 \%$ & $1.094 \%$ & $1.376 \%$ & $1.245 \%$ & $1.135 \%$ & $1.270 \%$ & $1.140 \%$ & $1.144 \%$ & $1.287 \%$ \\
\hline & Student-t & -1.611 & $-2.025^{*}$ & 0.949 & 1.829 & 0.397 & 0.964 & -0.260 & $2.186^{*}$ & 0.981 & -0.106 & -0.483 & -0.344 & -0.576 & -1.666 \\
\hline & Sign & -1.5 & -3.5 & 3.5 & 4.5 & 14.5 & $19.5^{*}$ & 0.5 & $24^{*}$ & 13 & 4 & 2 & 1 & 3 & 2 \\
\hline & $\begin{array}{l}\text { Signed Rank } \\
\text { \% Positive }\end{array}$ & -920.5 & -1254 & 725 & 873 & 840 & $1926^{*}$ & 36 & $3561.5^{*}$ & 1413.5 & 99.5 & 376.5 & -187.5 & -441.5 & -1086.5 \\
\hline & Days & $49.76 \%$ & $48.34 \%$ & $51.66 \%$ & $52.13 \%$ & $56.87 \% *$ & $59.24 \% *$ & $50.24 \%$ & $61.43 \% *$ & 56.19\%* & $51.90 \%$ & $50.95 \%$ & $50.48 \%$ & $51.43 \%$ & $50.95 \%$ \\
\hline \multirow{8}{*}{$\begin{array}{l}1989 \\
\text { to } \\
1998\end{array}$} & \# of Obs. & 73 & 73 & 73 & 73 & 73 & 73 & 73 & 72 & 72 & 72 & 72 & 72 & 72 & 72 \\
\hline & Mean & $-0.012 \%$ & $0.037 \%$ & $0.024 \%$ & $0.034 \%$ & $0.084 \%$ & $0.153 \%$ & $-0.141 \%$ & $0.263 \%$ & $0.140 \%$ & $0.076 \%$ & $-0.017 \%$ & $0.110 \%$ & $-0.139 \%$ & $0.059 \%$ \\
\hline & Median & $0.000 \%$ & $-0.120 \%$ & $0.110 \%$ & $0.116 \%$ & $0.137 \%$ & $0.179 \%$ & $0.021 \%$ & $0.217 \%$ & $0.117 \%$ & $0.084 \%$ & $-0.088 \%$ & $0.075 \%$ & $-0.040 \%$ & $0.059 \%$ \\
\hline & Std deviation & $0.687 \%$ & $0.871 \%$ & $1.056 \%$ & $0.852 \%$ & $0.777 \%$ & $0.804 \%$ & $1.130 \%$ & $0.936 \%$ & $0.885 \%$ & $0.716 \%$ & $0.755 \%$ & $0.949 \%$ & $0.872 \%$ & $0.752 \%$ \\
\hline & Student-t & -0.153 & 0.365 & 0.193 & 0.340 & 0.922 & 1.622 & -1.065 & $2.384 *$ & 1.342 & 0.899 & -0.186 & 0.986 & -1.348 & 0.667 \\
\hline & Sign & 0.5 & -2.5 & 6.5 & 1.5 & 8.5 & 8.5 & 0.5 & 8 & 6 & 7 & -7 & 4 & -1 & 8 \\
\hline & $\begin{array}{l}\text { Signed Rank } \\
\text { \% Positive }\end{array}$ & 41 & -24.5 & 300.5 & 91.5 & 319.5 & $380.5 *$ & -34.5 & $452^{*}$ & $366^{*}$ & 202 & -71 & 121 & -134 & 220 \\
\hline & Days & $52.05 \%$ & $46.58 \%$ & $58.90 \%$ & $52.05 \%$ & $61.64 \% *$ & $61.64 \% *$ & $50.68 \%$ & $61.11 \% *$ & $58.33 \%$ & $59.72 \% *$ & $40.28 \% *$ & $55.56 \%$ & $48.61 \%$ & $61.11 \% *$ \\
\hline \multirow{8}{*}{$\begin{array}{l}1999 \\
\text { to } \\
2010\end{array}$} & \# of Obs. & 138 & 138 & 138 & 138 & 138 & 138 & 138 & 138 & 138 & 138 & 138 & 138 & 138 & 138 \\
\hline & Mean & $-0.208 \%$ & $-0.326 \%$ & $0.129 \%$ & $0.232 \%$ & $0.000 \%$ & $0.056 \%$ & $0.045 \%$ & $0.179 \%$ & $0.055 \%$ & $-0.052 \%$ & $-0.056 \%$ & $-0.099 \%$ & $0.003 \%$ & $-0.256 \%$ \\
\hline & Median & $-0.111 \%$ & $-0.015 \%$ & $-0.056 \%$ & $0.024 \%$ & $0.057 \%$ & $0.081 \%$ & $0.001 \%$ & $0.357 \%$ & $0.110 \%$ & $-0.064 \%$ & $0.142 \%$ & $-0.069 \%$ & $0.040 \%$ & $-0.088 \%$ \\
\hline & Std deviation & $1.478 \%$ & $1.646 \%$ & $1.581 \%$ & $1.482 \%$ & $1.179 \%$ & $1.560 \%$ & $1.073 \%$ & $1.559 \%$ & $1.399 \%$ & $1.301 \%$ & $1.471 \%$ & $1.225 \%$ & $1.263 \%$ & $1.483 \%$ \\
\hline & Student-t & -1.651 & $-2.323^{*}$ & 0.960 & 1.842 & -0.002 & 0.420 & 0.488 & 1.346 & 0.463 & -0.471 & -0.446 & -0.945 & 0.029 & $-2.028 *$ \\
\hline & Sign & -2 & -1 & -3 & 3 & 6 & 11 & 0 & $16^{*}$ & 7 & -3 & 9 & -3 & 4 & -6 \\
\hline & $\begin{array}{l}\text { Signed Rank } \\
\% \text { Positive }\end{array}$ & -659.5 & -755.5 & 5.5 & 476.5 & 9.5 & 615.5 & 119.5 & $1455.5^{*}$ & 285.5 & -227.5 & 238.5 & -328.5 & -53.5 & $-1012.5^{*}$ \\
\hline & Days & $48.55 \%$ & $49.28 \%$ & $47.83 \%$ & $52.17 \%$ & $54.35 \%$ & $57.97 \% *$ & $50.00 \%$ & $61.59 \% *$ & $55.07 \%$ & $47.83 \%$ & $56.52 \%$ & $47.83 \%$ & $52.90 \%$ & $45.65 \% *$ \\
\hline \multirow{8}{*}{$\begin{array}{l}2007 \\
\text { to } \\
2010\end{array}$} & \# of Obs. & 42 & 42 & 42 & 42 & 42 & 42 & 42 & 42 & 42 & 42 & 42 & 42 & 42 & 42 \\
\hline & Mean & $-0.300 \%$ & $-0.667 \%$ & $0.188 \%$ & $0.605 \%$ & $0.053 \%$ & $-0.081 \%$ & $-0.118 \%$ & $0.034 \%$ & $0.198 \%$ & $-0.326 \%$ & $-0.434 \%$ & $-0.159 \%$ & $0.231 \%$ & $-0.278 \%$ \\
\hline & Median & $0.125 \%$ & $-0.074 \%$ & $-0.130 \%$ & $0.073 \%$ & $-0.053 \%$ & $0.005 \%$ & $-0.105 \%$ & $0.426 \%$ & $0.173 \%$ & $-0.079 \%$ & $0.215 \%$ & $0.100 \%$ & $0.138 \%$ & $0.043 \%$ \\
\hline & Std deviation & $2.075 \%$ & $2.313 \%$ & $2.436 \%$ & $2.195 \%$ & $1.284 \%$ & $2.206 \%$ & $1.425 \%$ & $2.180 \%$ & $1.679 \%$ & $1.550 \%$ & $1.935 \%$ & $1.715 \%$ & $1.509 \%$ & $2.206 \%$ \\
\hline & Student-t & -0.938 & -1.869 & 0.500 & 1.786 & 0.266 & -0.239 & -0.536 & 0.101 & 0.765 & -1.365 & -1.454 & -0.601 & 0.993 & -0.818 \\
\hline & Sign & 1 & -1 & -3 & 4 & -1 & 1 & -2 & 5 & 4 & -2 & 4 & 3 & 3 & 1 \\
\hline & $\begin{array}{l}\text { Signed Rank } \\
\% \text { Positive }\end{array}$ & -71.5 & -83.5 & -56.5 & 120.5 & 26.5 & 29.5 & -55.5 & 110.5 & 49.5 & -94.5 & -52.5 & -1.5 & 74.5 & -47.5 \\
\hline & Days & $52.38 \%$ & $47.62 \%$ & $42.86 \%$ & $59.52 \%$ & $47.62 \%$ & $52.38 \%$ & $45.24 \%$ & $61.90 \%$ & $59.52 \%$ & $45.24 \%$ & $59.52 \%$ & $57.14 \%$ & $57.14 \%$ & $52.38 \%$ \\
\hline
\end{tabular}




\section{Table 10: NASDAQ Industrial Daily Rates of Return}

The daily rates of return are shown for the first seven and the last seven trading days of the month. The first seven trading days are depicted as positive numbers where 1 is the first trading day and the last seven trading days are depicted by negative numbers where -1 is the last day. The rates of return are calculated as the log of the ratio of the previous day and the current day. Student-t as well as the non-parametric tests - sign, signed rank and binomial proportions-is also reported. $*$ - significance at the $95 \%$ level

\begin{tabular}{|c|c|c|c|c|c|c|c|c|c|c|c|c|c|c|c|}
\hline & Trading Day & -7 & -6 & -5 & -4 & -3 & -2 & -1 & 1 & 2 & 3 & 4 & 5 & 6 & 7 \\
\hline 1989 & \# of Obs. & 236 & 236 & 236 & 237 & 237 & 237 & 237 & 236 & 236 & 236 & 236 & 236 & 236 & 236 \\
\hline to & Mean & $-0.126 \%$ & $-0.137 \%$ & $-0.021 \%$ & $0.197 \%$ & $-0.005 \%$ & $0.229 \%$ & $0.254 \%$ & $0.071 \%$ & $0.146 \%$ & $0.055 \%$ & $-0.008 \%$ & $-0.045 \%$ & $-0.076 \%$ & $-0.112 \%$ \\
\hline \multirow[t]{6}{*}{2010} & Median & $0.043 \%$ & $-0.016 \%$ & $0.092 \%$ & $0.095 \%$ & $0.173 \%$ & $0.251 \%$ & $0.281 \%$ & $0.248 \%$ & $0.204 \%$ & $0.037 \%$ & $0.096 \%$ & $0.017 \%$ & $0.076 \%$ & $-0.027 \%$ \\
\hline & Std deviation & $1.488 \%$ & $1.360 \%$ & $1.449 \%$ & $1.549 \%$ & $1.422 \%$ & $1.598 \%$ & $1.280 \%$ & $1.726 \%$ & $1.646 \%$ & $1.535 \%$ & $1.500 \%$ & $1.372 \%$ & $1.516 \%$ & $1.527 \%$ \\
\hline & Student-t & -1.298 & -1.546 & -0.221 & 1.958 & -0.049 & $2.210^{*}$ & $3.051^{*}$ & 0.629 & 1.359 & 0.555 & -0.081 & -0.510 & -0.773 & -1.127 \\
\hline & Sign & 3.5 & -2 & 8.5 & 10.5 & $16.5^{*}$ & $28.5^{*}$ & $29 *$ & $21 *$ & $24 *$ & 3 & 13 & 1 & 6.5 & -2 \\
\hline & Signed Rank & -466 & -754 & 141 & 1594.5 & 1190.5 & $3770.5^{*}$ & $4455^{*}$ & $2378^{*}$ & $2109 *$ & 525 & 705 & -355 & 116 & -1054 \\
\hline & $\%$ Positive Days & $51.69 \%$ & $49.15 \%$ & $53.81 \%$ & $54.43 \%$ & $56.96 \% *$ & $62.03 \% *$ & $62.45 \% *$ & $58.90 \% *$ & $60.17 \% *$ & $51.27 \%$ & $55.51 \%$ & $50.42 \%$ & $52.97 \%$ & $49.15 \%$ \\
\hline \multirow{8}{*}{$\begin{array}{l}1989 \\
\text { to } \\
1998\end{array}$} & \# of Obs. & 98 & 98 & 99 & 99 & 99 & 99 & 99 & 98 & 98 & 98 & 98 & 98 & 98 & 98 \\
\hline & Mean & $0.028 \%$ & $0.035 \%$ & $-0.092 \%$ & $-0.001 \%$ & $0.103 \%$ & $0.330 \%$ & $0.304 \%$ & $0.112 \%$ & $0.193 \%$ & $0.120 \%$ & $0.000 \%$ & $0.015 \%$ & $-0.098 \%$ & $0.063 \%$ \\
\hline & Median & $0.125 \%$ & $0.023 \%$ & $0.010 \%$ & $0.027 \%$ & $0.202 \%$ & $0.329 \%$ & $0.442 \%$ & $0.202 \%$ & $0.231 \%$ & $0.179 \%$ & $0.080 \%$ & $-0.007 \%$ & $0.081 \%$ & $-0.004 \%$ \\
\hline & Std deviation & $0.886 \%$ & $0.889 \%$ & $1.155 \%$ & $0.960 \%$ & $1.030 \%$ & $0.891 \%$ & $1.086 \%$ & $1.041 \%$ & $1.042 \%$ & $0.983 \%$ & $0.889 \%$ & $1.036 \%$ & $1.011 \%$ & $0.959 \%$ \\
\hline & Student-t & 0.317 & 0.387 & -0.793 & -0.006 & 0.999 & $3.684^{*}$ & $2.787^{*}$ & 1.064 & 1.832 & 1.212 & -0.003 & 0.141 & -0.963 & 0.654 \\
\hline & Sign & 5.5 & 1 & 1 & 1.5 & 8.5 & $21.5^{*}$ & $23^{*}$ & 7 & $16^{*}$ & 8 & 7 & 0 & 6 & 0 \\
\hline & Signed R & 205.5 & 69.5 & -7.5 & 80 & 523 & 1198* & $1491.5^{*}$ & 427.5 & $598.5^{*}$ & 459.5 & 139.5 & 12.5 & 164.5 & 150.5 \\
\hline & $\%$ Positive Days & $56.12 \%$ & $51.02 \%$ & $51.52 \%$ & $51.52 \%$ & $58.59 \% *$ & $71.72 \% *$ & $73.74 \% *$ & $57.14 \%$ & $66.33 \% *$ & $58.16 \%$ & $57.14 \%$ & $50.00 \%$ & $56.12 \%$ & $50.00 \%$ \\
\hline \multirow{8}{*}{$\begin{array}{l}1999 \\
\text { to } \\
2010\end{array}$} & $\#$ of Obs. & 138 & 138 & 137 & 138 & 138 & 138 & 138 & 138 & 138 & 138 & 138 & 138 & 138 & 138 \\
\hline & Mean & $-0.235 \%$ & $-0.259 \%$ & $0.031 \%$ & $0.339 \%$ & $-0.082 \%$ & $0.157 \%$ & $0.217 \%$ & $0.041 \%$ & $0.112 \%$ & $0.009 \%$ & $-0.013 \%$ & $-0.088 \%$ & $-0.061 \%$ & $-0.237 \%$ \\
\hline & Median & $-0.049 \%$ & $-0.024 \%$ & $0.130 \%$ & $0.129 \%$ & $0.137 \%$ & $0.200 \%$ & $0.112 \%$ & $0.333 \%$ & $0.164 \%$ & $-0.110 \%$ & $0.155 \%$ & $0.033 \%$ & $0.000 \%$ & $-0.114 \%$ \\
\hline & Std deviation & $1.793 \%$ & $1.605 \%$ & $1.632 \%$ & $1.850 \%$ & $1.646 \%$ & $1.953 \%$ & $1.406 \%$ & $2.084 \%$ & $1.969 \%$ & $1.831 \%$ & $1.817 \%$ & $1.569 \%$ & $1.794 \%$ & $1.820 \%$ \\
\hline & Student-t & -1.541 & -1.894 & 0.219 & $2.150 *$ & -0.585 & 0.945 & 1.817 & 0.233 & 0.669 & 0.060 & -0.086 & -0.661 & -0.397 & -1.527 \\
\hline & Sign & -2 & -3 & 7.5 & 9 & 8 & 7 & 6 & $14^{*}$ & 8 & -5 & 6 & 1 & 0.5 & -2 \\
\hline & Signed Rank & -512.5 & -542.5 & 69.5 & 787.5 & 24.5 & 698.5 & 750.5 & 766.5 & 458.5 & -169.5 & 187.5 & -214.5 & -53.5 & -770.5 \\
\hline & $\%$ Positive Days & $48.55 \%$ & $47.83 \%$ & $55.47 \%$ & $56.52 \%$ & $55.80 \%$ & $55.07 \%$ & $54.35 \% *$ & $60.14 \% *$ & $55.80 \%$ & $46.38 \%$ & $54.35 \%$ & $50.72 \%$ & $50.72 \%$ & $48.55 \%$ \\
\hline \multirow{8}{*}{$\begin{array}{l}2007 \\
\text { to } \\
2010\end{array}$} & $\#$ of Obs. & 42 & 42 & 42 & 42 & 42 & 42 & 42 & 42 & 42 & 42 & 42 & 42 & 42 & 42 \\
\hline & Mean & $-0.317 \%$ & $-0.282 \%$ & $-0.175 \%$ & $0.674 \%$ & $0.101 \%$ & $-0.061 \%$ & $-0.026 \%$ & $0.029 \%$ & $0.156 \%$ & $-0.373 \%$ & $-0.454 \%$ & $-0.099 \%$ & $0.136 \%$ & $-0.173 \%$ \\
\hline & Median & $0.109 \%$ & $-0.059 \%$ & $-0.047 \%$ & $0.275 \%$ & $0.284 \%$ & $-0.007 \%$ & $-0.034 \%$ & $0.264 \%$ & $0.194 \%$ & $-0.186 \%$ & $0.107 \%$ & $0.160 \%$ & $0.072 \%$ & $0.118 \%$ \\
\hline & Std deviation & $2.278 \%$ & $1.629 \%$ & $1.537 \%$ & $2.354 \%$ & $1.513 \%$ & $2.380 \%$ & $1.407 \%$ & $2.480 \%$ & $1.927 \%$ & $1.711 \%$ & $1.978 \%$ & $1.885 \%$ & $1.638 \%$ & $2.185 \%$ \\
\hline & Student- $t$ & -0.903 & -1.123 & -0.740 & 1.854 & 0.435 & -0.165 & -0.122 & 0.075 & 0.526 & -1.413 & -1.489 & -0.339 & 0.539 & -0.512 \\
\hline & Sign & 1 & -2 & -1 & 4 & 3 & 0 & -1 & 6 & 4 & -3 & 2 & 3 & 0 & 3 \\
\hline & Signed Rank & -60.5 & -54.5 & -70.5 & 130.5 & 48.5 & 17.5 & -31.5 & 87.5 & 64.5 & -96.5 & -62.5 & 10.5 & 41.5 & -21.5 \\
\hline & \% Positive Days & $52.38 \%$ & $45.24 \%$ & $47.62 \%$ & $59.52 \%$ & $57.14 \%$ & $50.00 \%$ & $47.62 \%$ & $64.29 \%$ & $59.52 \%$ & $42.86 \%$ & $54.76 \%$ & $57.14 \%$ & $50.00 \%$ & $57.14 \%$ \\
\hline
\end{tabular}




\section{Table 11: NASDAQ Transportation Daily Rates of Return}

The daily rates of return are shown for the first seven and the last seven trading days of the month. The first seven trading days are depicted as positive numbers where 1 is the first trading day and the last seven trading days are depicted by negative numbers where -1 is the last day. The rates of return are calculated as the log of the ratio of the previous day

and the current day. Student-t as well as the non-parametric tests - sign, signed rank and binomial proportions-is also reported. * - significance at the $95 \%$ level

\begin{tabular}{|c|c|c|c|c|c|c|c|c|c|c|c|c|c|c|c|}
\hline & Trading Day & -7 & -6 & -5 & -4 & -3 & -2 & -1 & 1 & 2 & 3 & 4 & 5 & 6 & 7 \\
\hline 1989 & \# of Obs. & 236 & 236 & 237 & 237 & 237 & 237 & 237 & 236 & 236 & 236 & 236 & 236 & 236 & 236 \\
\hline to & Mean & $-0.167 \%$ & $-0.111 \%$ & $-0.049 \%$ & $0.171 \%$ & $0.066 \%$ & $0.259 \%$ & $0.319 \%$ & $0.085 \%$ & $0.205 \%$ & $0.089 \%$ & $-0.006 \%$ & $-0.129 \%$ & $-0.169 \%$ & $-0.101 \%$ \\
\hline \multirow{6}{*}{2010} & Median & $-0.083 \%$ & $-0.063 \%$ & $-0.061 \%$ & $0.075 \%$ & $0.090 \%$ & $0.158 \%$ & $0.319 \%$ & $0.193 \%$ & $0.178 \%$ & $0.113 \%$ & $0.009 \%$ & $-0.073 \%$ & $-0.095 \%$ & $-0.051 \%$ \\
\hline & Std deviation & $1.649 \%$ & $1.283 \%$ & $1.198 \%$ & $1.478 \%$ & $1.335 \%$ & $1.422 \%$ & $1.260 \%$ & $1.552 \%$ & $1.630 \%$ & $1.341 \%$ & $1.324 \%$ & $1.519 \%$ & $1.631 \%$ & $1.494 \%$ \\
\hline & Student-t & -1.553 & -1.332 & -0.633 & 1.786 & 0.756 & $2.800 *$ & $3.903 *$ & 0.841 & 1.929 & 1.014 & -0.074 & -1.302 & -1.593 & -1.037 \\
\hline & Sign & -9 & -4 & -7 & 11.5 & 7 & 13.5 & $29.5^{*}$ & $19.5^{*}$ & $16^{*}$ & 11 & 1 & -8 & -12 & -6 \\
\hline & $\begin{array}{l}\text { Signed Rank } \\
\% \text { Positive }\end{array}$ & -1649 & -822 & -658 & 1646.5 & 941 & $3294.5^{*}$ & $4363.5^{*}$ & $2211^{*}$ & $2692^{*}$ & 1291 & 483 & -1189 & -1770 & -1253 \\
\hline & Days & $46.19 \%$ & $48.31 \%$ & $47.26 \%$ & $54.85 \%$ & $53.16 \%$ & $55.70 \% *$ & $62.45 \% *$ & $58.47 \% *$ & $56.78 \% *$ & $54.66 \%$ & $50.42 \%$ & $46.61 \%$ & $44.92 \%$ & $47.46 \%$ \\
\hline \multirow{8}{*}{$\begin{array}{l}1989 \\
\text { to } \\
1998\end{array}$} & \# of Obs. & 98 & 98 & 99 & 99 & 99 & 99 & 99 & 98 & 98 & 98 & 98 & 98 & 98 & 98 \\
\hline & Mean & $-0.104 \%$ & $0.039 \%$ & $-0.115 \%$ & $-0.030 \%$ & $0.060 \%$ & $0.301 \%$ & $0.397 \%$ & $0.076 \%$ & $0.278 \%$ & $0.159 \%$ & $0.001 \%$ & $-0.103 \%$ & $-0.229 \%$ & $-0.077 \%$ \\
\hline & Median & $0.054 \%$ & $0.010 \%$ & $-0.093 \%$ & $0.010 \%$ & $0.053 \%$ & $0.181 \%$ & $0.445 \%$ & $0.106 \%$ & $0.269 \%$ & $0.145 \%$ & $-0.112 \%$ & $-0.198 \%$ & $-0.062 \%$ & $-0.032 \%$ \\
\hline & Std deviation & $0.907 \%$ & $0.843 \%$ & $0.916 \%$ & $0.945 \%$ & $0.949 \%$ & $0.885 \%$ & $0.970 \%$ & $0.967 \%$ & $0.928 \%$ & $0.900 \%$ & $0.776 \%$ & $1.070 \%$ & $1.042 \%$ & $0.914 \%$ \\
\hline & Student-t & -1.133 & 0.462 & -1.253 & -0.321 & 0.625 & $3.382 *$ & $4.072 *$ & 0.776 & $2.966 *$ & 1.752 & 0.015 & -0.957 & -2.174 & -0.834 \\
\hline & Sign & 1 & 1 & -7 & 0.5 & 2 & 9.5 & $23.5^{*}$ & 4 & $13 *$ & 10 & -5 & -8 & -4 & -1 \\
\hline & $\begin{array}{l}\text { Signed Rank } \\
\text { \% Positive }\end{array}$ & -165.5 & 87.5 & -298.5 & 9 & 199.5 & $872 *$ & $1454^{*}$ & 270.5 & $904.5^{*}$ & 463.5 & -92.5 & -454.5 & -390.5 & -216.5 \\
\hline & Days & $51.02 \%$ & $51.02 \%$ & $43.43 \%$ & $50.51 \%$ & $52.53 \%$ & $59.60 \% *$ & $73.74 \% *$ & $54.08 \%$ & $63.27 \% *$ & $60.20 \%$ & $44.90 \%$ & $41.84 \%$ & $45.92 \%$ & $48.98 \%$ \\
\hline \multirow{8}{*}{$\begin{array}{l}1999 \\
\text { to } \\
2010\end{array}$} & \# of Obs. & 138 & 138 & 138 & 138 & 138 & 138 & 138 & 138 & 138 & 138 & 138 & 138 & 138 & 138 \\
\hline & Mean & $-0.211 \%$ & $-0.218 \%$ & $-0.002 \%$ & $0.316 \%$ & $0.070 \%$ & $0.228 \%$ & $0.264 \%$ & $0.091 \%$ & $0.153 \%$ & $0.038 \%$ & $-0.012 \%$ & $-0.147 \%$ & $-0.127 \%$ & $-0.118 \%$ \\
\hline & Median & $-0.190 \%$ & $-0.125 \%$ & $-0.004 \%$ & $0.184 \%$ & $0.133 \%$ & $0.135 \%$ & $0.091 \%$ & $0.334 \%$ & $0.036 \%$ & $0.028 \%$ & $0.143 \%$ & $0.006 \%$ & $-0.129 \%$ & $-0.065 \%$ \\
\hline & Std deviation & $2.019 \%$ & $1.514 \%$ & $1.367 \%$ & $1.753 \%$ & $1.558 \%$ & $1.709 \%$ & $1.433 \%$ & $1.863 \%$ & $1.986 \%$ & $1.583 \%$ & $1.606 \%$ & $1.774 \%$ & $1.947 \%$ & $1.798 \%$ \\
\hline & Student-t & -1.229 & -1.693 & -0.016 & $2.120^{*}$ & 0.527 & 1.569 & $2.162^{*}$ & 0.577 & 0.903 & 0.285 & -0.086 & -0.972 & -0.764 & -0.769 \\
\hline & Sign & -10 & -5 & 0 & 11 & 5 & 4 & 6 & $15.5^{*}$ & 3 & 1 & 6 & 0 & -8 & -5 \\
\hline & $\begin{array}{l}\text { Signed Rank } \\
\text { \% Positive }\end{array}$ & -671.5 & -579.5 & -51.5 & 899.5 & 252.5 & 836.5 & 717.5 & 787.5 & 454.5 & 178.5 & 278.5 & -151.5 & -437.5 & -444.5 \\
\hline & Days & $42.75 \% *$ & $46.38 \%$ & $50.00 \%$ & $57.97 \% *$ & $53.62 \% *$ & $52.90 \%$ & $54.35 \%$ & $61.59 \% *$ & $52.17 \%$ & $50.72 \%$ & $54.35 \%$ & $50.00 \%$ & $44.20 \%$ & $46.38 \%$ \\
\hline \multirow{8}{*}{$\begin{array}{l}2007 \\
\text { to } \\
2010\end{array}$} & \# of Obs. & 42 & 42 & 42 & 42 & 42 & 42 & 42 & 42 & 42 & 42 & 42 & 42 & 42 & 42 \\
\hline & Mean & $-0.416 \%$ & $-0.654 \%$ & $-0.256 \%$ & $0.453 \%$ & $0.101 \%$ & $-0.019 \%$ & $0.357 \%$ & $0.023 \%$ & $0.227 \%$ & $-0.297 \%$ & $-0.615 \%$ & $-0.215 \%$ & $0.305 \%$ & $0.003 \%$ \\
\hline & Median & $-0.391 \%$ & $-0.404 \%$ & $-0.322 \%$ & $0.048 \%$ & $0.190 \%$ & $0.122 \%$ & $0.004 \%$ & $0.499 \%$ & $0.358 \%$ & $-0.254 \%$ & $-0.161 \%$ & $0.211 \%$ & $0.063 \%$ & $-0.106 \%$ \\
\hline & Std deviation & $3.039 \%$ & $1.959 \%$ & $1.614 \%$ & $2.622 \%$ & $1.863 \%$ & $2.425 \%$ & $1.841 \%$ & $2.765 \%$ & $2.790 \%$ & $2.119 \%$ & $2.052 \%$ & $2.653 \%$ & $2.050 \%$ & $2.633 \%$ \\
\hline & Student-t & -0.887 & -2.162 & -1.027 & 1.121 & 0.352 & -0.052 & 1.255 & 0.053 & 0.528 & -0.909 & -1.943 & -0.524 & 0.963 & 0.008 \\
\hline & Sign & -4 & -4 & -2 & 0 & 0 & 2 & 0 & 7* & 3 & -3 & -4 & 2 & 0 & -1 \\
\hline & $\begin{array}{l}\text { Signed Rank } \\
\% \text { Positive }\end{array}$ & -90.5 & -151.5 & -136.5 & 37.5 & 18.5 & 15.5 & 62.5 & 88.5 & 58.5 & -82.5 & -118.5 & 6.5 & 55.5 & -17.5 \\
\hline & Days & $40.48 \%$ & $40.48 \%$ & $45.24 \%$ & $50.00 \%$ & $50.00 \%$ & $54.76 \%$ & $50.00 \%$ & $66.67 \% *$ & $57.14 \%$ & $42.86 \%$ & $40.48 \%$ & $54.76 \%$ & $50.00 \%$ & $47.62 \%$ \\
\hline
\end{tabular}


Table 12: Dow Jones Industrial Average, Dow Jones Transportation, Dow Jones Utilities, NASDAQ Industrial, NASDAQ Transportation SemiMonthly Rates of Returns

The semi-monthly rates of return are shown for the first and the second half of the month. A trading month is considered to have twenty days so the first half of the trading month consists of the first ten trading days and the second half consists of the last ten trading days for the month. Returns for each half month are calculated using the log of the ratio of the last trading day in that half to the first trading day in that half of the month.Student-t as well as the non-parametric tests - sign, signed rank and binomial proportions-is also reported. * - significance at the $95 \%$ level.

\begin{tabular}{|c|c|c|c|c|c|c|c|c|c|c|c|}
\hline & & \multicolumn{3}{|c|}{ DJIA } & \multicolumn{2}{|l|}{ DJT } & \multirow{2}{*}{$\frac{\text { DJU }}{2^{\text {nd }} \text { half }}$} & \multicolumn{2}{|c|}{ NASDAQ IND } & \multicolumn{2}{|c|}{ NASDAQ TRANS } \\
\hline & & $1^{\text {st }}$ half & $2^{\text {nd }}$ half & $1^{\text {st }}$ half & $2^{\text {nd }}$ half & $1^{\text {st }}$ half & & $1^{\text {st }}$ half & $2^{\text {nd }}$ half & $1^{\text {st }}$ half & $2^{\text {nd }}$ half \\
\hline \multirow{8}{*}{$\begin{array}{l}1989 \\
\text { to } \\
2010\end{array}$} & \# of Obs. & 257 & 258 & 257 & 258 & 257 & 258 & 235 & 236 & 235 & 236 \\
\hline & Mean & 0.002 & -0.002 & $0.106 \%$ & $-0.093 \%$ & -0.004 & 0.004 & $0.177 \%$ & $0.254 \%$ & $0.085 \%$ & $0.253 \%$ \\
\hline & Median & 0.005 & 0.001 & $0.131 \%$ & $0.282 \%$ & -0.002 & 0.006 & $0.748 \%$ & $0.770 \%$ & $0.192 \%$ & $0.641 \%$ \\
\hline & Std deviation & 0.029 & 0.029 & $3.885 \%$ & $4.233 \%$ & 0.033 & 0.028 & $4.590 \%$ & $4.484 \%$ & $3.853 \%$ & $4.260 \%$ \\
\hline & Student-t & 1.108 & -1.203 & 0.436 & -0.351 & -1.910 & $2.095^{*}$ & 0.592 & 0.870 & 0.337 & 0.911 \\
\hline & Sign & $22.5^{*}$ & 6 & 2.5 & 9 & -5 & $31 *$ & 12.5 & $19 *$ & 4.5 & $17 *$ \\
\hline & Signed Rank & $2553.5^{*}$ & -587.5 & 1081.5 & 338.5 & -1227 & $4071.5^{*}$ & 1526 & $2245^{*}$ & 745 & 1890 \\
\hline & \% Positive Days & $58.75 \% *$ & $52.33 \%$ & $50.97 \%$ & $53.49 \%$ & $48.25 \%$ & $62.02 \% *$ & $55.32 \%$ & $58.05 \% *$ & $51.91 \%$ & $57.20 \% *$ \\
\hline \multirow{8}{*}{$\begin{array}{l}1989 \\
\text { to } \\
1998\end{array}$} & \# of Obs. & 120 & 120 & 120 & 120 & 120 & 120 & 98 & 98 & 98 & 98 \\
\hline & Mean & 0.005 & 0.000 & $0.426 \%$ & $0.054 \%$ & -0.001 & 0.004 & $0.604 \%$ & $0.528 \%$ & $0.283 \%$ & $0.379 \%$ \\
\hline & Median & 0.009 & 0.001 & $0.398 \%$ & $0.449 \%$ & -0.002 & 0.006 & $0.840 \%$ & $0.968 \%$ & $0.173 \%$ & $0.726 \%$ \\
\hline & Std deviation & 0.025 & 0.028 & $3.473 \%$ & $3.633 \%$ & 0.024 & 0.022 & $3.131 \%$ & $4.122 \%$ & $2.607 \%$ & $3.697 \%$ \\
\hline & Student-t & $2.284^{*}$ & 0.146 & 1.344 & 0.162 & -0.616 & 1.799 & $1.911^{*}$ & 1.268 & 1.075 & 1.014 \\
\hline & Sign & $16^{*}$ & 5 & 4 & 7 & -2.5 & $16^{*}$ & $11 *$ & $15^{*}$ & 4 & 10 \\
\hline & Signed Rank & 1039* & 224 & 636 & 234 & -45 & $879 *$ & $579.5^{*}$ & $704.5^{*}$ & 356.5 & 537.5 \\
\hline & \% Positive Days & $63.33 \% *$ & $54.17 \%$ & $53.33 \%$ & $55.83 \%$ & $48.33 \%$ & $63.33 \% *$ & $61.22 \% *$ & $65.31 \% *$ & $54.08 \%$ & $60.20 \% *$ \\
\hline \multirow{8}{*}{$\begin{array}{l}1999 \\
\text { to } \\
2010\end{array}$} & $\#$ of Obs. & 137 & 138 & 137 & 138 & 137 & 138 & 137 & 138 & 137 & 138 \\
\hline & Mean & -0.001 & -0.004 & $-0.175 \%$ & $-0.220 \%$ & -0.006 & 0.004 & $-0.129 \%$ & $0.059 \%$ & $-0.057 \%$ & $0.163 \%$ \\
\hline & Median & 0.003 & 0.000 & $-0.162 \%$ & $0.134 \%$ & -0.002 & 0.008 & $0.272 \%$ & $0.340 \%$ & $0.206 \%$ & $0.623 \%$ \\
\hline & Std deviation & 0.032 & 0.030 & $4.206 \%$ & $4.703 \%$ & 0.040 & 0.032 & $5.388 \%$ & $4.729 \%$ & $4.544 \%$ & $4.629 \%$ \\
\hline & Student-t & -0.290 & -1.728 & -0.488 & -0.549 & -1.844 & 1.344 & -0.279 & 0.147 & -0.147 & 0.413 \\
\hline & Sign & 6.5 & 1 & -1.5 & 2 & -2.5 & $15^{*}$ & 1.5 & 4 & 0.5 & 7 \\
\hline & Signed Rank & 201.5 & -555.5 & -135.5 & -65.5 & -555.5 & $1152.5^{*}$ & 149.5 & 380.5 & 0.5 & 388.5 \\
\hline & \% Positive Days & $54.74 \%$ & $50.72 \%$ & $48.91 \%$ & $51.45 \%$ & $48.18 \%$ & $60.87 \% *$ & $51.09 \%$ & $52.90 \%$ & $50.36 \%$ & $55.07 \%$ \\
\hline \multirow{8}{*}{$\begin{array}{l}2007 \\
\text { to } \\
2010\end{array}$} & $\#$ of Obs. & 42 & 42 & 42 & 42 & 42 & 42 & 42 & 42 & 42 & 42 \\
\hline & Mean & -0.005 & -0.004 & $-0.104 \%$ & $-0.620 \%$ & -0.006 & -0.005 & $-0.534 \%$ & $-0.168 \%$ & $-0.439 \%$ & $-0.747 \%$ \\
\hline & Median & 0.002 & 0.005 & $0.442 \%$ & $0.250 \%$ & -0.001 & -0.001 & $-0.225 \%$ & $0.422 \%$ & $-1.146 \%$ & $-0.227 \%$ \\
\hline & Std deviation & 0.042 & 0.031 & $5.571 \%$ & $4.857 \%$ & 0.034 & 0.030 & $5.908 \%$ & $3.851 \%$ & $6.087 \%$ & $5.274 \%$ \\
\hline & Student-t & -0.758 & -0.737 & -0.121 & -0.827 & -1.084 & -1.013 & -0.586 & -0.282 & -0.468 & -0.919 \\
\hline & Sign & 2 & 3 & 2 & 1 & 0 & -1 & -1 & 1 & -2 & -1 \\
\hline & Signed Rank & -11.5 & -0.5 & 4.5 & -35.5 & -55.5 & -22.5 & -6.5 & 14.5 & -25.5 & -79.5 \\
\hline & \% Positive Days & $54.76 \%$ & $57.14 \%$ & $54.76 \%$ & $52.38 \%$ & $50.00 \%$ & $47.62 \%$ & $47.62 \%$ & $52.38 \%$ & $45.24 \%$ & $47.62 \%$ \\
\hline
\end{tabular}




\section{Table 13: Dow Jones Industrial Average, Dow Jones Transportation, Dow Jones Utilities, NASDAQ Industrial, NASDAQ Transportation}

\section{Weekly Rates of Returns}

The weekly rates of return are shown for the first and the last trading week of the month. A trading month is considered to have twenty days so the first week of the trading month consists of the first five trading days and the lastweek consists of the last five trading days. Returns for each week are calculated using the log of the ratio of the last trading day in that week to the first trading day in that trading week. Student-t as well as the non-parametric tests - sign, signed rank and binomial proportions-is also reported. * - significance at the $95 \%$ leve

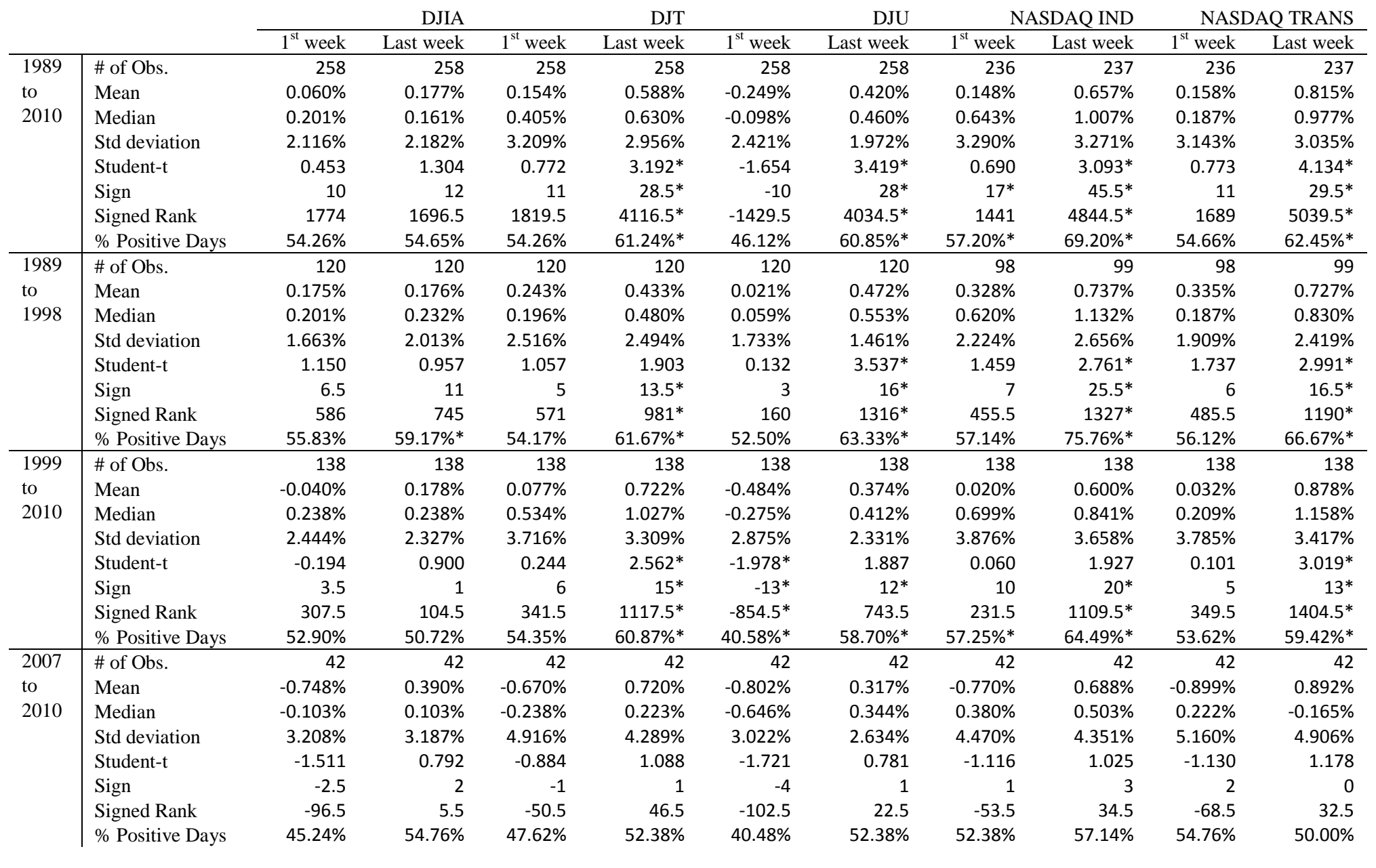


Table 14: Dow Jones Industrial Average, Dow Jones Transportation, Dow Jones Utilities, NASDAQ Industrial, NASDAQ Transportation Three Day Rates of Returns The three day rates of return are shown for the first and the last three days of the month. Returns for each three day period are calculated using the log of the ratio of the third trading day to the first trading day in that three day period. Student-t as well as the non-parametric tests - sign, signed rank and binomial proportions-is also reported. * significance at the $95 \%$ level

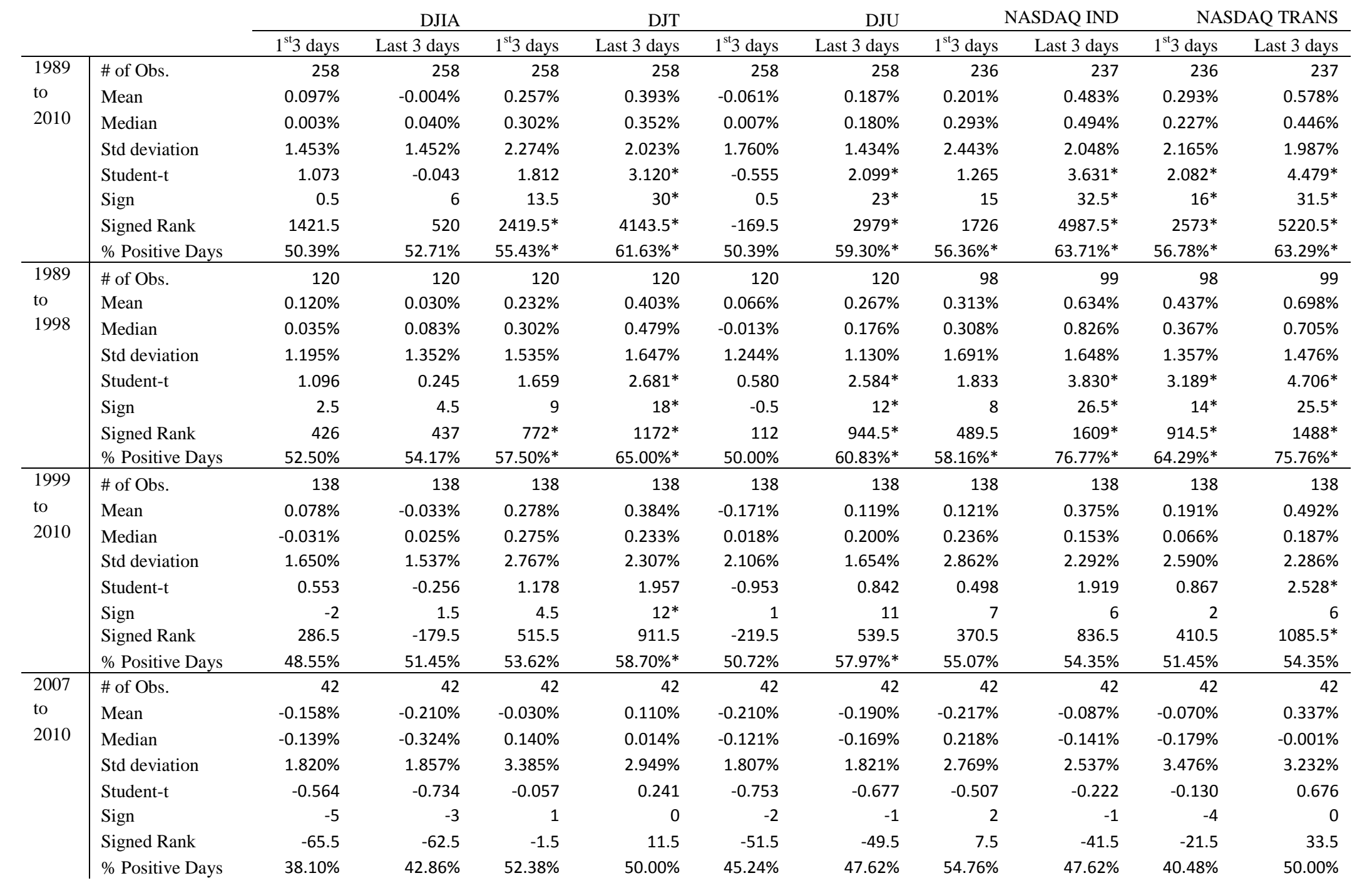




\section{Table 15: NASDAQ, NASDAQ100, S\&P500, Russell 3000, Russell 2000 and Russell 1000Semi-Monthly Rates of Returns}

The semi-monthly rates of return are shown for the first and the second half of the month. A trading month is considered to have twenty days so the first half of the trading month consists of the first ten trading days and the second half consists of the last ten trading days for the month. Returns for each half month are calculated using the log of the ratio of the last trading day in that half to the first trading day in that half of the month.Student-t as well as the non-parametric tests - sign, signed rank and binomial proportions-is also reported. * - significance at the $95 \%$ level

\begin{tabular}{|c|c|c|c|c|c|c|c|c|c|c|c|c|c|}
\hline & \multicolumn{2}{|c|}{ NASDAQ } & \multicolumn{2}{|c|}{ NASDAQ100 } & \multicolumn{2}{|c|}{ SP500 } & \multicolumn{2}{|c|}{ RUSSELL 3000} & \multicolumn{2}{|c|}{ RUSSELL 2000} & \multicolumn{2}{|c|}{ RUSSELL 1000} \\
\hline & & $1^{\text {st }}$ half & $2^{\text {nd }}$ half & $1^{\text {st }}$ half & $2^{\text {nd }}$ half & $1^{\text {st }}$ half & $2^{\text {nd }}$ half & $1^{\text {st }}$ half & $2^{\text {nd }}$ half & $1^{\text {st }}$ half & $2^{\text {nd }}$ half & $1^{\text {st }}$ half & $2^{\text {nd }}$ half \\
\hline \multirow{8}{*}{$\begin{array}{l}1989 \\
\text { to } \\
2010\end{array}$} & \# of Obs. & 257 & 258 & 257 & 258 & 257 & 258 & 257 & 258 & 257 & 258 & 209 & 211 \\
\hline & Mean & $0.260 \%$ & $-0.009 \%$ & $0.373 \%$ & $-0.060 \%$ & $0.049 \%$ & $-0.090 \%$ & $0.060 \%$ & $-0.026 \%$ & $0.036 \%$ & $0.263 \%$ & $0.014 \%$ & $-0.153 \%$ \\
\hline & Median & $0.587 \%$ & $0.750 \%$ & $0.788 \%$ & $0.519 \%$ & $0.406 \%$ & $0.268 \%$ & $0.356 \%$ & $0.384 \%$ & $0.419 \%$ & $0.527 \%$ & $0.370 \%$ & $0.294 \%$ \\
\hline & Std deviation & $4.513 \%$ & $4.251 \%$ & $5.093 \%$ & $4.808 \%$ & $3.002 \%$ & $2.805 \%$ & $3.062 \%$ & $2.878 \%$ & $3.778 \%$ & $3.705 \%$ & $3.153 \%$ & $2.917 \%$ \\
\hline & Student-t & 0.924 & -0.032 & 1.175 & -0.200 & 0.263 & -0.516 & 0.313 & -0.146 & 0.154 & 1.142 & 0.062 & -0.764 \\
\hline & Sign & 15.5 & $18^{*}$ & $16.5^{*}$ & $18^{*}$ & $16.5^{*}$ & $16^{*}$ & $21.5^{*}$ & $22 *$ & 14.5 & $20 *$ & $15^{*}$ & $17.5^{*}$ \\
\hline & Signed Rank & $2507.5^{*}$ & $1511.5^{*}$ & $2531.5^{*}$ & 1039.5 & $1624.5^{*}$ & 584.5 & $1720.5^{*}$ & 1198.5 & 1386.5 & $2895.5^{*}$ & $1001^{*}$ & $406 *$ \\
\hline & $\%$ Positive Days & $56.03 \% *$ & $56.98 \% *$ & $56.42 \% *$ & $56.98 \% *$ & $56.42 \% *$ & $56.20 \% *$ & $58.37 \% *$ & $58.53 \%$ & $55.64 \% *$ & $57.75 \% *$ & $57.42 \% *$ & $58.29 \% *$ \\
\hline \multirow{8}{*}{$\begin{array}{l}1989 \\
\text { to } \\
1998\end{array}$} & \# of Obs. & 120 & 120 & 120 & 120 & 120 & 120 & 120 & 120 & 120 & 120 & 72 & 73 \\
\hline & Mean & $0.664 \%$ & $0.456 \%$ & $0.786 \%$ & $0.647 \%$ & $0.423 \%$ & $0.230 \%$ & $0.426 \%$ & $0.248 \%$ & $0.389 \%$ & $0.294 \%$ & $0.582 \%$ & $0.135 \%$ \\
\hline & Median & $0.826 \%$ & $1.067 \%$ & $0.938 \%$ & $0.736 \%$ & $0.717 \%$ & $0.461 \%$ & $0.761 \%$ & $0.628 \%$ & $0.610 \%$ & $0.780 \%$ & $0.751 \%$ & $0.529 \%$ \\
\hline & Std deviation & $3.143 \%$ & $3.685 \%$ & $4.055 \%$ & $4.221 \%$ & $2.427 \%$ & $2.624 \%$ & $2.392 \%$ & $2.693 \%$ & $2.663 \%$ & $3.475 \%$ & $2.279 \%$ & $2.772 \%$ \\
\hline & Student-t & $2.314^{*}$ & 1.355 & $2.123^{*}$ & 1.680 & 1.908 & 0.960 & 1.952 & 1.010 & 1.599 & 0.928 & $2.166^{*}$ & 0.417 \\
\hline & Sign & $13^{*}$ & $15^{*}$ & 11 & $17 *$ & $15 *$ & $12^{*}$ & $19 *$ & $15^{*}$ & $16^{*}$ & $17^{*}$ & $12 *$ & $11.5^{*}$ \\
\hline & Signed Rank & $1096 *$ & 925* & $954^{*}$ & 886* & $909 *$ & $649 *$ & $943^{*}$ & $730 *$ & $910^{*}$ & $787^{*}$ & $455^{*}$ & 298.5 \\
\hline & $\%$ Positive Days & $60.83 \% *$ & $62.50 \% *$ & $59.17 \% *$ & $64.17 \% *$ & $62.50 \% *$ & $60.00 \% *$ & $65.83 \% *$ & $62.50 \% *$ & $63.33 \% *$ & $64.17 \% *$ & $66.67 \% *$ & $65.75 \% *$ \\
\hline \multirow{8}{*}{$\begin{array}{l}1999 \\
\text { to } \\
2010\end{array}$} & \# of Obs. & 137 & 138 & 137 & 138 & 137 & 138 & 137 & 138 & 137 & 138 & 137 & 138 \\
\hline & Mean & $-0.093 \%$ & $-0.412 \%$ & $0.012 \%$ & $-0.674 \%$ & $-0.278 \%$ & $-0.369 \%$ & $-0.261 \%$ & $-0.265 \%$ & $-0.272 \%$ & $0.236 \%$ & $-0.285 \%$ & $-0.306 \%$ \\
\hline & Median & $0.341 \%$ & $0.248 \%$ & $0.630 \%$ & $0.118 \%$ & $0.140 \%$ & $0.091 \%$ & $0.202 \%$ & $0.229 \%$ & $-0.278 \%$ & $0.193 \%$ & $0.200 \%$ & $0.176 \%$ \\
\hline & Std deviation & $5.425 \%$ & $4.664 \%$ & $5.844 \%$ & $5.203 \%$ & $3.403 \%$ & $2.934 \%$ & $3.525 \%$ & $3.019 \%$ & $4.524 \%$ & $3.907 \%$ & $3.498 \%$ & $2.990 \%$ \\
\hline & Student-t & -0.201 & -1.038 & 0.023 & -1.523 & -0.956 & -1.476 & -0.868 & -1.030 & -0.705 & 0.711 & -0.954 & -1.203 \\
\hline & Sign & 2.5 & 3 & 5.5 & 1 & 1.5 & 4 & 2.5 & 7 & -1.5 & 3 & 3 & 6 \\
\hline & Signed Rank & 206.5 & -172.5 & 293.5 & -406.5 & -134.5 & -389.5 & -92.5 & -152.5 & -127.5 & 611.5 & -116 & -233.5 \\
\hline & \% Positive Days & $51.82 \%$ & $52.17 \%$ & $54.01 \%$ & $50.72 \%$ & $51.09 \%$ & $52.90 \%$ & $51.82 \%$ & $55.07 \%$ & $48.91 \%$ & $52.17 \%$ & $52.55 \%$ & $54.35 \%$ \\
\hline \multirow{8}{*}{$\begin{array}{l}2007 \\
\text { to } \\
2010\end{array}$} & \# of Obs. & 42 & 42 & 42 & 42 & 42 & 42 & 42 & 42 & 42 & 42 & 42 & 42 \\
\hline & Mean & $-0.265 \%$ & $-0.258 \%$ & $-0.101 \%$ & $-0.116 \%$ & $-0.487 \%$ & $-0.445 \%$ & $-0.431 \%$ & $-0.426 \%$ & $-0.450 \%$ & $-0.476 \%$ & $-0.470 \%$ & $-0.422 \%$ \\
\hline & Median & $0.577 \%$ & $0.584 \%$ & $0.673 \%$ & $0.401 \%$ & $0.219 \%$ & $0.450 \%$ & $0.338 \%$ & $0.463 \%$ & $-0.306 \%$ & $0.417 \%$ & $0.290 \%$ & $0.539 \%$ \\
\hline & Std deviation & $5.189 \%$ & $3.616 \%$ & $5.240 \%$ & $3.715 \%$ & $4.630 \%$ & $3.191 \%$ & $4.783 \%$ & $3.300 \%$ & $5.853 \%$ & $4.268 \%$ & $4.749 \%$ & $3.249 \%$ \\
\hline & Student-t & -0.331 & -0.463 & -0.124 & -0.202 & -0.681 & -0.904 & -0.584 & -0.836 & -0.498 & -0.722 & -0.642 & -0.842 \\
\hline & Sign & 1 & 2 & 2 & 1 & 2 & 4 & 3 & 5 & -1 & 2 & 3 & 5 \\
\hline & Signed Rank & 10.5 & 13.5 & 22.5 & 21.5 & -10.5 & -14.5 & -4.5 & -8.5 & -18.5 & -1.5 & -7.5 & -12.5 \\
\hline & \% Positive Days & $52.38 \%$ & $54.76 \%$ & $54.76 \%$ & $52.38 \%$ & $54.76 \%$ & $59.52 \%$ & $57.14 \%$ & $61.90 \%$ & $47.62 \%$ & $54.76 \%$ & $57.14 \%$ & $61.90 \%$ \\
\hline
\end{tabular}




\section{Table 16: NASDAQ, NASDAQ100, S\&P500, Russell 3000, Russell 2000 and Russell 1000 Weekly Rates of Returns}

The weekly rates of return are shown for the first and the last trading week of the month. A trading month is considered to have twenty days so the first week of the trading month consists of the first five trading days and the lastweek consists of the last five trading days. Returns for each week are calculated using the log of the ratio of the last trading day in that week to the first trading day in that trading week. Student-t as well as the non-parametric tests - sign, signed rank and binomial proportions-is also reported. $*$ - significance at the $95 \%$ level

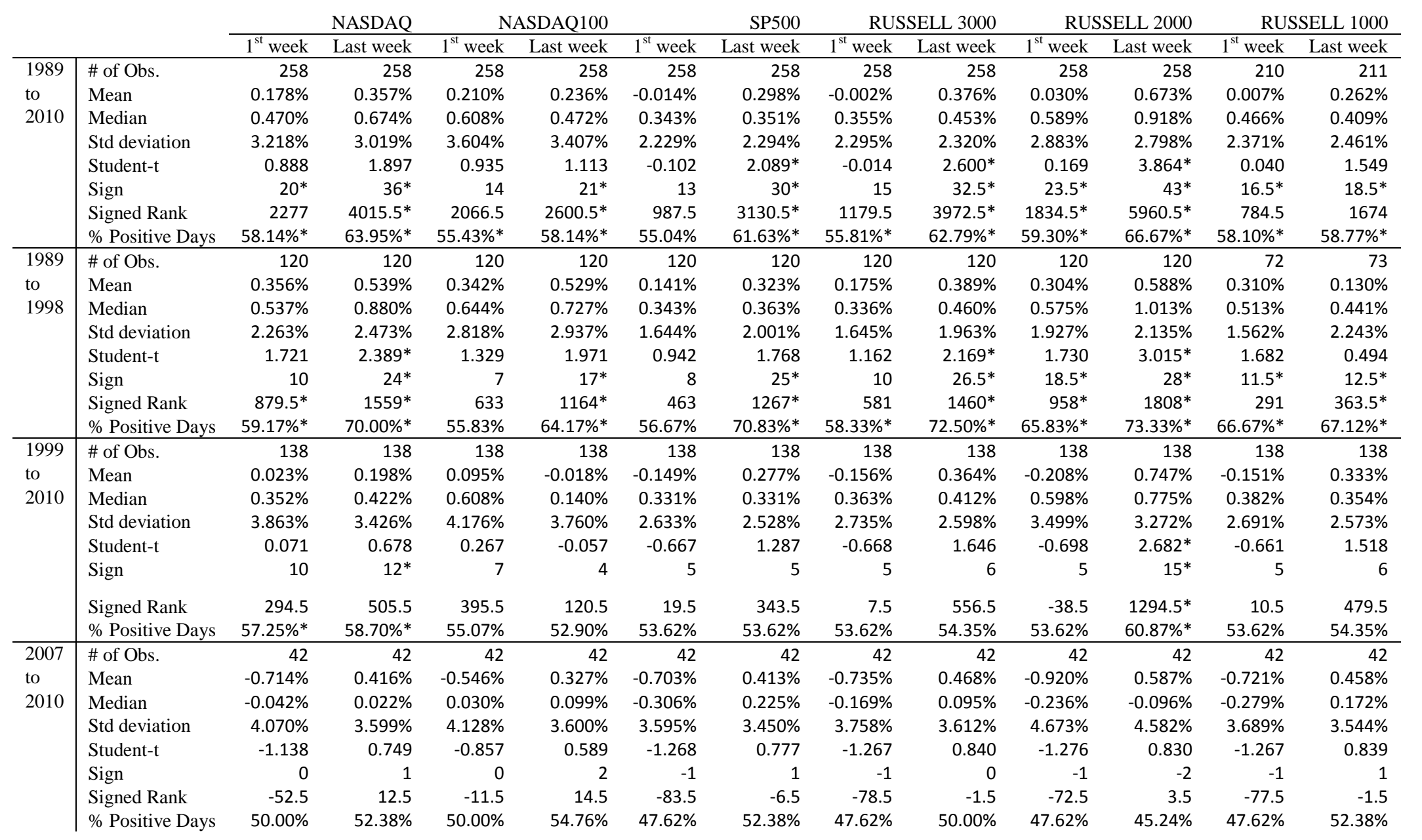




\section{Table 17: NASDAQ, NASDAQ100, S\&P500, Russell 3000, Russell 2000 and Russell 1000 Three Day Rates of Returns}

The three day rates of return are shown for the first and the last three days of the month. Returns for each three day period are calculated using the log of the ratio of the third trading day to the first trading day in that three day period. Student-t as well as the non-parametric tests - sign, signed rank and binomial proportions-is also reported. * significance at the $95 \%$ level

\begin{tabular}{|c|c|c|c|c|c|c|c|c|c|c|c|c|c|}
\hline & \multicolumn{2}{|r|}{ NASDAQ } & \multicolumn{2}{|c|}{ NASDAQ100 } & \multicolumn{2}{|r|}{ SP500 } & \multicolumn{2}{|c|}{ RUSSELL 3000} & \multicolumn{2}{|c|}{ RUSSELL 2000} & \multicolumn{2}{|c|}{ RUSSELL 1000} \\
\hline & & $1^{\text {st } 3 \text { days }}$ & Last 3 days & $1^{\text {st } 3 \text { days }}$ & Last 3 days & $1^{\text {st } 3 \text { days }}$ & Last 3 days & $1^{\text {st } 3 \text { days }}$ & Last 3 days & $1^{\text {st } 3 \text { days }}$ & Last 3 days & $1^{\text {st } 3 \text { days }}$ & Last 3 days \\
\hline \multirow{8}{*}{$\begin{array}{l}1989 \\
\text { to } \\
2010\end{array}$} & \# of Obs. & 258 & 258 & 258 & 258 & 258 & 258 & 258 & 258 & 258 & 258 & 210 & 211 \\
\hline & Mean & $0.198 \%$ & $0.223 \%$ & $0.218 \%$ & $0.113 \%$ & $0.053 \%$ & $0.096 \%$ & $0.072 \%$ & $0.168 \%$ & $0.088 \%$ & $0.423 \%$ & $0.076 \%$ & $0.070 \%$ \\
\hline & Median & $0.291 \%$ & $0.271 \%$ & $0.253 \%$ & $0.102 \%$ & $0.066 \%$ & $0.146 \%$ & $0.113 \%$ & $0.219 \%$ & $0.288 \%$ & $0.609 \%$ & $0.144 \%$ & $0.126 \%$ \\
\hline & Std deviation & $2.481 \%$ & $1.988 \%$ & $2.945 \%$ & $2.340 \%$ & $1.593 \%$ & $1.559 \%$ & $1.630 \%$ & $1.552 \%$ & $1.955 \%$ & $1.819 \%$ & $1.702 \%$ & $1.660 \%$ \\
\hline & Student-t & 1.282 & 1.801 & 1.189 & 0.772 & 0.530 & 0.989 & 0.713 & 1.742 & 0.726 & $3.739 *$ & 0.647 & 0.610 \\
\hline & Sign & 11 & $17 *$ & 7 & 9 & 7 & $19 *$ & 9 & $25 *$ & $25^{*}$ & 39* & 8.5 & 11.5 \\
\hline & Signed Rank & 2056.5 & $3740.5^{*}$ & 1657.5 & 1889.5 & 1013.5 & 2306.5 & 1416.5 & $3685.5^{*}$ & $2484.5^{*}$ & $5864.5^{*}$ & 992.5 & 1263 \\
\hline & $\%$ Positive Days & $54.26 \%$ & $56.59 \% *$ & $52.71 \%$ & $53.49 \%$ & $52.71 \%$ & $57.36 \% *$ & $53.49 \%$ & $59.69 \% *$ & $59.69 \% *$ & $65.12 \% *$ & $54.29 \%$ & $55.45 \%$ \\
\hline \multirow{8}{*}{$\begin{array}{l}1989 \\
\text { to } \\
1998\end{array}$} & \# of Obs. & 120 & 120 & 120 & 120 & 120 & 120 & 120 & 120 & 120 & 120 & 72 & 73 \\
\hline & Mean & $0.286 \%$ & $0.411 \%$ & $0.240 \%$ & $0.292 \%$ & $0.115 \%$ & $0.152 \%$ & $0.158 \%$ & $0.228 \%$ & $0.259 \%$ & $0.563 \%$ & $0.216 \%$ & $0.012 \%$ \\
\hline & Median & $0.348 \%$ & $0.577 \%$ & $0.280 \%$ & $0.369 \%$ & $0.115 \%$ & $0.212 \%$ & $0.104 \%$ & $0.273 \%$ & $0.310 \%$ & $0.714 \%$ & $0.266 \%$ & $0.151 \%$ \\
\hline & Std deviation & $1.588 \%$ & $1.632 \%$ & $2.067 \%$ & $2.113 \%$ & $1.193 \%$ & $1.382 \%$ & $1.182 \%$ & $1.321 \%$ & $1.298 \%$ & $1.281 \%$ & $1.188 \%$ & $1.556 \%$ \\
\hline & Student-t & $1.972 *$ & $2.758^{*}$ & 1.273 & 1.515 & 1.054 & 1.205 & 1.463 & 1.892 & $2.183^{*}$ & $4.812^{*}$ & 1.543 & 0.065 \\
\hline & Sign & 10 & $20 *$ & 4 & $16^{*}$ & 2 & $16^{*}$ & 4 & $19 *$ & $17^{*}$ & $37 *$ & 3 & 6.5 \\
\hline & Signed Rank & 891* & $1835^{*}$ & 516 & 1023* & 359 & $968^{*}$ & 526 & $1428 *$ & 1131* & $2317^{*}$ & 276 & 243.5 \\
\hline & \% Positive Days & $58.33 \% *$ & $66.67 \% *$ & $53.33 \%$ & $63.33 \% *$ & $51.67 \%$ & $63.33 \% *$ & $53.33 \%$ & $65.83 \% *$ & $64.17 \% *$ & $80.83 \% *$ & $54.17 \%$ & $58.90 \%$ \\
\hline \multirow{8}{*}{$\begin{array}{l}1999 \\
\text { to } \\
2010\end{array}$} & \# of Obs. & 138 & 138 & 138 & 138 & 138 & 138 & 138 & 138 & 138 & 138 & 138 & 138 \\
\hline & Mean & $0.122 \%$ & $0.060 \%$ & $0.199 \%$ & $-0.044 \%$ & $-0.002 \%$ & $0.047 \%$ & $-0.002 \%$ & $0.116 \%$ & $-0.060 \%$ & $0.302 \%$ & $0.003 \%$ & $0.100 \%$ \\
\hline & Median & $0.018 \%$ & $-0.075 \%$ & $0.223 \%$ & $-0.157 \%$ & $0.051 \%$ & $0.045 \%$ & $0.143 \%$ & $0.124 \%$ & $0.213 \%$ & $0.108 \%$ & $0.112 \%$ & $0.076 \%$ \\
\hline & Std deviation & $3.057 \%$ & $2.246 \%$ & $3.544 \%$ & $2.518 \%$ & $1.876 \%$ & $1.702 \%$ & $1.938 \%$ & $1.731 \%$ & $2.379 \%$ & $2.179 \%$ & $1.916 \%$ & $1.717 \%$ \\
\hline & Student-t & 0.468 & 0.311 & 0.658 & -0.204 & -0.010 & 0.327 & -0.012 & 0.789 & -0.295 & 1.629 & 0.018 & 0.687 \\
\hline & Sign & 1 & -3 & 3 & -7 & 5 & 3 & 5 & 6 & 8 & 2 & 5.5 & 5 \\
\hline & Signed Rank & 202.5 & 107.5 & 326.5 & -120.5 & 118.5 & 201.5 & 160.5 & 478.5 & 145.5 & 749.5 & 148.5 & 426.5 \\
\hline & \% Positive Days & $50.72 \%$ & $47.83 \%$ & $52.17 \%$ & $44.93 \%$ & $53.62 \%$ & $52.17 \%$ & $53.62 \%$ & $54.35 \%$ & $55.80 \%$ & $51.45 \%$ & $54.35 \%$ & $53.62 \%$ \\
\hline \multirow{8}{*}{$\begin{array}{l}2007 \\
\text { to } \\
2010\end{array}$} & \# of Obs. & 42 & 42 & 42 & 42 & 42 & 42 & 42 & 42 & 42 & 42 & 42 & 42 \\
\hline & Mean & $-0.110 \%$ & $-0.257 \%$ & $0.015 \%$ & $-0.353 \%$ & $-0.125 \%$ & $-0.235 \%$ & $-0.143 \%$ & $-0.203 \%$ & $-0.311 \%$ & $-0.215 \%$ & $-0.128 \%$ & $-0.199 \%$ \\
\hline & Median & $0.199 \%$ & $-0.220 \%$ & $0.250 \%$ & $-0.245 \%$ & $0.041 \%$ & $-0.215 \%$ & $0.084 \%$ & $-0.116 \%$ & $0.083 \%$ & $-0.550 \%$ & $0.084 \%$ & $-0.175 \%$ \\
\hline & Std deviation & $2.422 \%$ & $2.297 \%$ & $2.498 \%$ & $2.237 \%$ & $2.135 \%$ & $2.161 \%$ & $2.252 \%$ & $2.253 \%$ & $2.986 \%$ & $2.972 \%$ & $2.200 \%$ & $2.206 \%$ \\
\hline & Student-t & -0.293 & -0.726 & 0.040 & -1.021 & -0.380 & -0.705 & -0.411 & -0.583 & -0.675 & -0.469 & -0.377 & -0.585 \\
\hline & Sign & 3 & -4 & 3 & -2 & 1 & -2 & 2 & -2 & 1 & -6 & 2 & -2 \\
\hline & Signed Rank & 20.5 & -54.5 & 42.5 & -65.5 & -22.5 & -45.5 & -13.5 & -43.5 & -19.5 & -90.5 & -11.5 & -39.5 \\
\hline & \% Positive Days & $57.14 \%$ & $40.48 \%$ & $57.14 \%$ & $45.24 \%$ & $52.38 \%$ & $45.24 \%$ & $54.76 \%$ & $45.24 \%$ & $52.38 \%$ & $35.71 \% *$ & $54.76 \%$ & $45.24 \%$ \\
\hline
\end{tabular}

